\title{
Robotic and laparoscopic sacrocolpopexy for pelvic organ prolapse: a systematic review and meta-analysis
}

\author{
Jiang Yang", Yong He", Xiaoyi Zhang, Zhi Wang, Xiaohu Zuo, Likun Gao, Li Hong \\ Department of Obstetrics and Gynecology, Renmin Hospital of Wuhan University, Wuhan, China \\ Contributions: (I) Conception and design: J Yang, L Hong; (II) Administrative support: L Hong; (III) Provision of study materials or patients: J \\ Yang, Y He; (IV) Collection and assembly of data: Y He, X Zhang, Z Wang, X Zuo, L Gao; (V) Data analysis and interpretation: J Yang, Y He; (VI) \\ Manuscript writing: All authors; (VII) Final approval of manuscript: All authors. \\ "These authors contributed equally to this work \\ Correspondence to: Prof. Li Hong. Department of Obstetrics and Gynecology, Renmin Hospital of Wuhan University, 238 Jiefang Road, Wuhan \\ 430060, China. Email: drhongli77@gmail.com.
}

Background: Sacrocolpopexy is the gold standard procedure for treating pelvic organ prolapse (POP) patients with apical defects. Different surgical approaches have emerged and been utilized successively, including traditional laparoscopy, single-hole laparoscopy, robotic laparoscopy, vaginal-assisted laparoscopy, and transvaginal approaches. Robotic sacrocolpopexy (RSC) has attracted increasing attention as an emerging surgical technique and has unique advantages, such as a "simulated wrist" mechanical arm and high-definition three-dimensional (3D) visual field, which has gradually begun to be utilized in the clinical setting.

Methods: We followed the Preferred Reporting Items for Systematic reviews and Meta-Analyses (PRISMA) reporting checklist, and a systematic literature search was conducted on six databases from their inception to 1st March 2020. We evaluated patients with POP who underwent RSC or laparoscopic sacrocolpopexy (LSC), outcomes (including perioperative outcomes: blood loss, operating times, blood transfusion, and hospital stay), surgery-related complications, as well as cure and recurrence rates.

Results: A total of 49 articles were available, including 3,014 patients, among which 18 were comparative studies on LSC vs. RSC, and 31 were non-comparative single-arm studies on RSC. For RSC, median operative time was 226 [90-604] minutes, estimated blood loss was 56 [5-1,500] mL, and hospital stay was 1.55 [1-16] days. Intraoperative complications and postoperative complications occurred in 74 (2.7\%) and $360(13.0 \%)$ patients, respectively. Of 2,768 RSC patients, 40 had been converted from a robotassisted approach to other approaches, and 134 of 1,852 patients (7.2\%) have recurrent prolapses of any compartment. Compared to LSC, RSC was associated with significantly lower blood loss and lower conversion rate. However, more operative time was observed in RSC. No significant differences were observed in perioperative transfusion, intraoperative and postoperative complications, or objective recurrence between RSC and LSC.

Conclusions: RSC's application seems to contribute some advantages compared to conventional laparoscopic surgery, although both approaches appear to promote equivalent clinical outcomes. Notably, heterogeneity among studies might have affected the outcome of the study. Consequently, high-quality and large-sample randomized trials comparing both techniques are necessitated.

Keywords: Pelvic organ prolapse (POP); robotic sacrocolpopexy (RSC); laparoscopic sacrocolpopexy (LSC); meta-analysis

Submitted May 29, 2020. Accepted for publication Nov 20, 2020.

doi: 10.21037/atm-20-4347

View this article at: http://dx.doi.org/10.21037/atm-20-4347 


\section{Introduction}

Pelvic organ prolapse (POP) is a gynecological disease group that includes uterine prolapse and anterior and posterior vaginal wall bulge. Studies have illustrated that approximately $30 \%$ of middle-aged and older women experience prolapse of different degrees (1), among whom $11-19 \%$ of POP patients undergo surgical treatment (2). Laparoscopic sacrocolpopexy (LSC) is one of the classic and effective surgical procedures for POP treatment (3). Based on the operational requirements, LSC's standard suture fixation site is the anterior longitudinal ligament on the pelvic surface of the S1 vertebral body, which attaches to the sacrococcygeal curve concavity posteriorly. The laparoscopic surgical field in this area is severely limited, and damage to the presacral vascular plexus causes uncontrollable bleeding.

Consequently, LSC operation has a higher risk and more complicated operation challenges (3). The robotic surgery system has obtained prominent clinical application potential with the advantages of three-dimensional (3D) magnification of the visual field, flexible operation in narrow spaces, and physiological vibration filtering. Moreover, numerous clinical centers have carried out robotic sacrocolpopexy (RSC). Although few studies have summarized and compared LSC and RSC, most have contained a small series of cases detailing RSC application over a relatively short time frame. Technical barriers for surgeons were present in the early applications of RSC. After the extensive application of RSC over recent years, a large number of surgeons have been trained, which may lead to a paradox compared to early results. In this study, we conducted a systematic review and meta-analysis by collating the relevant data of worldwide studies over recent years, exploring and comparing the clinical efficacy of LSC and RSC in order to further evaluate the application potential of RSC.

We present the following article following the Preferred Reporting Items for Systematic reviews and Meta-Analyses (PRISMA) reporting checklist (available at http://dx.doi. org/10.21037/atm-20-4347).

\section{Methods}

\section{Search strategy}

Two independent investigators searched the databases PubMed (Medline), Scopus, EMBASE, CNKI, WanFang
DATA, and the Cochrane Library systematically, using the terms 'sacrocolpopexy or sacral colpopexy', 'robotassisted sacrocolpopexy or robotic sacrocolpopexy', and 'laparoscopic sacrocolpopexy or robotic-assist laparoscopic sacropopexy'. The 'related articles' function was used to broaden the search, and all citations were considered relevant. The retrieval time was not limited, and the last iteration of the searching procedure was 1st March 2020.

\section{Criteria for considering studies for this review}

\section{Inclusion criteria}

All studies were reviewed carefully to make sure that they met the following inclusion criteria: (I) comparing the clinical efficacy of RSC and LSC and published before March 2020; (II) reported at least one essential outcome of RSC and LSC comparative data such as operation time, intraoperative bleeding volume, intraoperative complications, conversion, average hospital stay, POP cure (POP $\leq 1$ grade), postoperative complications, subjective recurrence, objective recurrence, and reoperation et al.; (III) LSC cases $\geq 5$ for each study; and (IV) in the case of duplicate data, the latest study or larger sample size was included.

\section{Exclusion criteria}

The exclusion criteria were as follows: (I) letters, editorials, review articles, case series; (II) RSC cases $\leq 5$; (III) insufficient data or unclear data reporting; (IV) absence of original data available for extraction; and (V) duplicate publications with the same unit or the same author. Any differences in opinion were resolved through discussion and in consultation with the first author.

\section{Types of studies}

Published controlled trials comparing RSC and LSC's clinical efficacy before March 2020 were eligible for inclusion for the meta-analysis. Non-comparative singlearm studies on RSC were included in the systematic review.

\section{Types of participants}

For the meta-analysis, women undergoing sacrocolpopexy (robotic or robot-assisted) for POP, for any reason, were eligible for inclusion. Women who were treated by singlearm RSC were included in the systematic review. 


\section{Types of interventions}

Trials comparing robotic or robot-assisted sacrocolpopexy used to treat POP $v s$. LSC were eligible for inclusion. Only interventions performed during, immediately before, or within the 24 hours before surgery were considered for this review, compared to LSC, the interventions that were considered in this meta-analysis were robotic or robotassisted sacrocolpopexy.

\section{Outcomes of interest}

Outcomes were used to compare RSC and LSC as follows: (I) intraoperative parameters, including operative time (minutes), blood loss ( $\mathrm{mL}$ ), conversion to other approaches, bladder injury, bowel injury, vascular injury, ureteral injury, and all intraoperative complications; (II) postoperative parameters, including the length of hospital stay, perioperative blood transfusion, anorectal dysfunction, dyspareunia, mesh erosion, and all postoperative complications; (III) POP cure (POP $\leq 1$ grade), recurrence and reoperation at 24 months. All data sets involved the most recent updates of information.

\section{Data extraction}

All data were extracted by two researchers independently. The extracted data included: name of the first author, year of publication, study design, number of participants in robotic and laparoscopic groups, preoperative characteristics (POP classification, history of hysterectomy, history of pop related surgery), intraoperative variables (urinary incontinence surgery, hysterectomy, operation time, intraoperative bleeding, conversion, bladder injury, intestinal injury, vascular injury, ureteral injury, and other complications), postoperative variables (length of stay, perioperative blood transfusion, anorectal dysfunction, dyspareunia, mesh erosion, and other complications), POP cure ( $\mathrm{POP} \leq 1$ grade), and recurrence and reoperation.

\section{Assessment of methodological quality}

For single-arm RSC, studies meeting the inclusion criteria were assessed by two independent reviewers for methodological validity before inclusion in the review using a standardized critical appraisal tool from the Joanna Briggs Institute (JBI) for case series (4). Any disagreements that arose between the reviewers were resolved through discussion. All studies, regardless of their methodological quality, underwent data extraction and synthesis. For the meta-analysis component, we used the quality evaluation tool of clinical intervention research, the methodological index for non-randomized studies (MINORS) developed by French surgeon Karem Slim (5) in 2003 on a comprehensive review of literature and consensus of experts. There were 12 items in total, each of which scored 0-2 points. A score of 0 indicated no report; 1 indicated insufficient information; and 2 indicated sufficient information. Twelve indexes evaluated the literature quality, and research with a quality score $\geq$ of 18 was included in the meta-analysis.

\section{Statistical analysis}

Statistical analysis was performed with Cochrane Review software [Review Manager (RevMan) version 5.3 for Windows] and Stata 12 (version 12.0, StataCorp., College Station, TX, USA). Weighted mean difference (WMD) was used for continuous variable data, and the odds ratio (OR) was calculated for dichotomous variables. Both WMD and OR were expressed by a $95 \%$ confidence interval $(95 \%$ $\mathrm{CI})$. The heterogeneity was presented by using $\mathrm{I}^{2}$ values. If $\mathrm{P}>0.1, \mathrm{I}^{2} \leq 50 \%$, it was considered that there was no obvious heterogeneity among the included studies, and the fixed effect model was used to calculate the combined statistics. If it was considered that there was heterogeneity ( $\mathrm{P} \leq 0.1$, $\left.\mathrm{I}^{2}>50 \%\right)$, the random effect model was used to calculate the combined statistics. A significant statistical difference was considered if $\mathrm{P}<0.05$. Mean, median, and the ratio was performed for the single-arm studies to act as simple descriptive analysis parameters.

\section{Results}

For the final analysis, a total of 49 articles were available, including 3,014 patients. Of these, 18 were comparative studies on LSC $v s$. RSC, and 31 were non-comparative single-arm studies on RSC. Study selection as a PRISMA flowchart is summarized in Figure 1.

\section{Outcomes of RSC}

\section{Perioperative outcomes}

A summary of perioperative results in the RSC series is presented in Table 1. Two thousand nine hundred and sixteen patients had undergone RSC from 2004 to 2020. Median operative time was 226 [90-604] minutes, estimated 


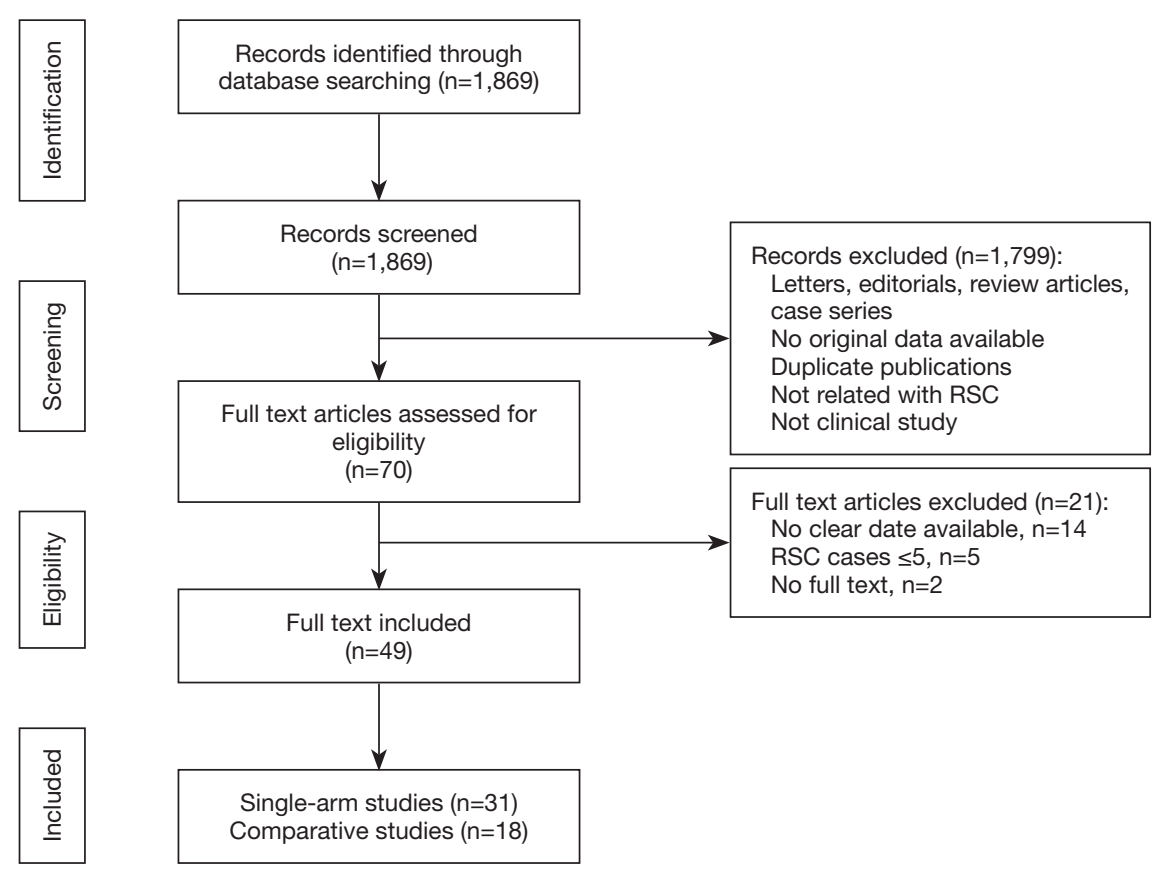

Figure 1 Flowchart of Preferred Reporting Items for Systematic reviews and Meta-Analyses (PRISMA) diagram. RSC, robotic sacrocolpopexy.

blood loss was $56[5-1,500] \mathrm{mL}$, and hospital stay was 1.55 [1-16] days. Of these patients, five had undergone blood transfusions.

Operative time reflects the surgical volume and execution of concomitant procedures and evaluates the surgeon's proficiency in performing RSC. We found that the operative time of RSC varied across different studies. In a large retrospective cohort study, Nosti et al. reported 262 RSCs with a median operative time of 316 [interquartile range (IQR): 109-604] minutes (27), and Ploumidis et al. reported 95 RSCs with a median operative time of 101 (IQR: 90120) minutes $(7,33)$. The difference among different institutions may be due to the execution of different concomitant procedures. RSC is inevitably combined with transobturator tape (TOT) implantation in patients with POP and stress urinary incontinence (SUI), and many patients opt for a concomitant hysterectomy during RSC. We observed that $21.7 \%$ and $25 \%$ of patients had performed hysterectomy and anti-incontinence procedures during RSC, respectively, leading to a great increase in operation time.

Blood loss is an important parameter to evaluate the quality of surgery. According to the results we observed, median blood loss was $56 \mathrm{~mL}$, and the least blood loss was $5 \mathrm{~mL}$ during RSC (44); as an attractive parameter to minimally invasive surgery, this minimal loss implied a hugely promising application of RSC. Although few studies have reported that RSC was also associated with a higher rate of estimated blood loss of $\geq 500 \mathrm{~mL}(7,27,29,44)$, previous laparotomy and subsequent adhesion formation may still be the underlying risk factor of higher blood loss, as opposed to the mode of the surgery itself (29). The hospital stay was 1-2 days for most participants; however, the hospital stay of one patient was 16 days due to syncopal crisis when standing up from postoperative day 1 , which was attributed to excessive tension in the posterior mesh. Robot-assisted laparoscopic reoperation was carried out to free posterior mesh and anchor it without tension. The ensuing postoperative course was uneventful, and she was discharged 14 days after the first surgical procedure (17).

The perioperative transfusion rate was $0.12 \%$ $(\mathrm{n}=5)(23,37,40,49,50)$. Blood loss caused by RSC was the cause of $40 \%(n=2)$ of transfusion; one case involved hemorrhage due to placement of retropubic midurethral sling, and the other was postoperative blood loss $(23,40)$. Blood loss caused by chronic disease accounted for $40 \%$ $(\mathrm{n}=2)$ of transfusion, including anemia secondary to chronic hemorrhoids in the postoperative period and transfusion recommended by hematology due to factor $\mathrm{V}$ Leiden deficiency combined with an estimated blood loss of $100 \mathrm{~mL}(37,50)$. 
Table 1 A summary of perioperative results in the RSC series

\begin{tabular}{|c|c|c|c|c|c|c|c|c|c|}
\hline Study & Institution & $\begin{array}{l}\text { Study } \\
\text { design }\end{array}$ & $\begin{array}{l}\text { Robotic } \\
\text { cases }\end{array}$ & $\begin{array}{l}\text { Concomitant } \\
\text { anti-incontinence } \\
\text { procedure } \\
\text { rate }(\%)\end{array}$ & $\begin{array}{l}\text { Concomitant } \\
\text { hysterectomy } \\
\text { rate }(\%)\end{array}$ & $\begin{array}{l}\text { Median/mean } \\
\text { operative time, } \\
\text { min }\end{array}$ & $\begin{array}{l}\text { Median/ } \\
\text { mean blood } \\
\text { loss, ml }\end{array}$ & $\begin{array}{l}\text { In-hospital } \\
\text { stay, d }\end{array}$ & $\begin{array}{l}\text { Transfusion } \\
\text { rate (\%) }\end{array}$ \\
\hline $\begin{array}{l}\text { Ferrando } \\
\text { et al. (6) }\end{array}$ & $\begin{array}{l}\text { Cleveland Clinic, } \\
\text { Cleveland, OH, USA }\end{array}$ & $\mathrm{RCT}$ & 24 & NA & NA & $214.2 \pm 51.5$ & NA & NA & NA \\
\hline $\begin{array}{l}\text { Elliott } \\
\text { et al. (8) }\end{array}$ & $\begin{array}{l}\text { Mayo Clinic, Rochester, } \\
\text { MN, USA }\end{array}$ & PS & 42 & $26(61.9)$ & NA & 186 [129-285] & $N A$ & NA & NA \\
\hline $\begin{array}{l}\text { Mueller } \\
\text { et al. (9) }\end{array}$ & $\begin{array}{l}\text { Loyola University } \\
\text { Chicago Stritch School } \\
\text { of Medicine, Chicago, IL, } \\
\text { USA }\end{array}$ & RCT & 40 & $22(55.0)$ & $21(52.5)$ & NA & NA & NA & NA \\
\hline $\begin{array}{l}\text { Shariati } \\
\text { et al. (11) }\end{array}$ & $\begin{array}{l}\text { UMDNJ New Jersey } \\
\text { Medical School, Newark, } \\
\text { NJ, USA }\end{array}$ & RS & 77 & NA & $3(3.8)$ & 273 [205-359] & $N A$ & $2[2-10]$ & $0(0.0)$ \\
\hline $\begin{array}{l}\text { Collins } \\
\text { et al. (12) }\end{array}$ & $\begin{array}{l}\text { University of Connecticut } \\
\text { Health Center, Hartford, } \\
\text { CT, USA }\end{array}$ & IPS & 30 & $9(30.0)$ & $21(70.0)$ & $262.8 \pm 51.8$ & $83.3 \pm 47$ & NA & NA \\
\hline $\begin{array}{l}\text { Elliott } \\
\text { et al. (13) }\end{array}$ & $\begin{array}{l}\text { Mayo Clinic, Rochester, } \\
\text { MN, USA }\end{array}$ & PS & 20 & $8(40.0)$ & NA & 192 [135-270] & NA & $1[1-2]$ & $0(0.0)$ \\
\hline $\begin{array}{l}\text { Chan } \\
\text { et al. (16) }\end{array}$ & $\begin{array}{l}\text { The Chinese University } \\
\text { of Hong Kong, Prince of } \\
\text { Wales Hospital, Hong } \\
\text { Kong, China }\end{array}$ & $\mathrm{RS}$ & 16 & $3(18.7)$ & NA & $230 \pm 42$ & $131.0 \pm 79.3$ & $7.5 \pm 7$ & $0(0.0)$ \\
\hline $\begin{array}{l}\text { Moreno } \\
\text { Sierra } \\
\text { et al. (17) }\end{array}$ & $\begin{array}{l}\text { Hospital Clinico San } \\
\text { Carlos, Universidad } \\
\text { Complutense, Madrid, } \\
\text { Spain }\end{array}$ & PS & 31 & NA & NA & 186 [150-230] & NA & $4.6[1-16]$ & $0(0.0)$ \\
\hline $\begin{array}{l}\text { Shimko } \\
\text { et al. (18) }\end{array}$ & $\begin{array}{l}\text { Mayo Clinic, Rochester, } \\
\text { MN, USA }\end{array}$ & RS & 40 & $24(60.0)$ & $0(0.0)$ & 186 [129-300] & NA & $1.2[1-7]$ & $0(0.0)$ \\
\hline $\begin{array}{l}\text { Linder } \\
\text { et al. (19) }\end{array}$ & $\begin{array}{l}\text { Mayo Clinic, Rochester, } \\
\text { MN, USA }\end{array}$ & PS & 84 & $55(65.4)$ & $0(0.0)$ & 160 [135-180] & 50 [25-100] & NA & NA \\
\hline $\begin{array}{l}\text { Elliott } \\
\text { et al. (20) }\end{array}$ & $\begin{array}{l}\text { Mayo Clinic, Rochester, } \\
\text { MN, USA }\end{array}$ & PS & 30 & $11(36.6)$ & NA & 186 & NA & $1[1-3]$ & $0(0.0)$ \\
\hline
\end{tabular}

Table 1 (continued) 
Table 1 (continued)

\begin{tabular}{|c|c|c|c|c|c|c|c|c|c|}
\hline Study & Institution & $\begin{array}{l}\text { Study } \\
\text { design }\end{array}$ & $\begin{array}{l}\text { Robotic } \\
\text { cases }\end{array}$ & $\begin{array}{l}\text { Concomitant } \\
\text { anti-incontinence } \\
\text { procedure } \\
\text { rate }(\%)\end{array}$ & $\begin{array}{l}\text { Concomitant } \\
\text { hysterectomy } \\
\text { rate }(\%)\end{array}$ & $\begin{array}{l}\text { Median/mean } \\
\text { operative time, } \\
\text { min }\end{array}$ & $\begin{array}{l}\text { Median/ } \\
\text { mean blood } \\
\text { loss, mL }\end{array}$ & $\begin{array}{l}\text { In-hospital } \\
\text { stay, d }\end{array}$ & $\begin{array}{l}\text { Transfusion } \\
\text { rate (\%) }\end{array}$ \\
\hline $\begin{array}{l}\text { Osmundsen } \\
\text { et al. (21) }\end{array}$ & $\begin{array}{l}\text { Oregon Health \& } \\
\text { Science University, } \\
\text { Portland, OR, USA }\end{array}$ & $\mathrm{RS}$ & 102 & NA & $45(44.1)$ & NA & NA & NA & NA \\
\hline $\begin{array}{l}\text { Pulliam } \\
\text { et al. (23) }\end{array}$ & $\begin{array}{l}\text { Massachusetts General } \\
\text { Hospital, Harvard } \\
\text { Medical School, Boston, } \\
\text { MA, USA }\end{array}$ & RS & 43 & 15 (34.8) & $4(9.3)$ & $242 \pm 54$ & $83 \pm 78$ & $1 \pm 0$ & $1(2.3)$ \\
\hline $\begin{array}{l}\text { Kenton } \\
\text { et al. (25) }\end{array}$ & $\begin{array}{l}\text { Northwestern University, } \\
\text { Feinberg School of } \\
\text { Medicine, Chicago, IL, } \\
\text { USA }\end{array}$ & RCT & 40 & NA & NA & NA & NA & NA & NA \\
\hline $\begin{array}{l}\text { Mueller } \\
\text { et al. (26) }\end{array}$ & $\begin{array}{l}\text { The Loyola University } \\
\text { Chicago, Stritch School } \\
\text { of Medicine, Maywood, } \\
\text { IL,USA }\end{array}$ & RS & 226 & $117(51.7)$ & $151(66.8)$ & $255 \pm 66$ & $99 \pm 74.3$ & NA & NA \\
\hline $\begin{array}{l}\text { Nosti } \\
\text { et al. (27) }\end{array}$ & $\begin{array}{l}\text { FPMRS Medstar } \\
\text { Washington Hospital } \\
\text { Center, Georgetown } \\
\text { University School of } \\
\text { Medicine, Washington, } \\
\text { DC, USA }\end{array}$ & RS & 262 & NA & NA & $316[109-604]$ & $\begin{array}{l}100 \\
{[10-1,000]}\end{array}$ & $1[1-16]$ & $0(0.0)$ \\
\hline $\begin{array}{l}\text { Unger } \\
\text { et al. (29) }\end{array}$ & $\begin{array}{l}\text { Cleveland Clinic, } \\
\text { Cleveland, } \mathrm{OH}, \mathrm{USA}\end{array}$ & RS & 121 & NA & NA & $275 \pm 56$ & NA & NA & NA \\
\hline $\begin{array}{l}\text { Biler } \\
\text { et al. (30) }\end{array}$ & $\begin{array}{l}\text { University of Health } \\
\text { Sciences Tepecik } \\
\text { Training and Research } \\
\text { Hospital, Izmir, Turkey }\end{array}$ & PS & 20 & $6(30.0)$ & NA & $217 \pm 40.9$ & $55 \pm 30$ & $5.1 \pm 1.1$ & $0(0.0)$ \\
\hline $\begin{array}{l}\text { Matthews } \\
\text { et al. (31) }\end{array}$ & $\begin{array}{l}\text { Virginia Commonwealth } \\
\text { University Medical } \\
\text { Center, Richmond, VA, } \\
\text { USA }\end{array}$ & PS & 85 & $39(45.8)$ & 37 (43.5) & $195 \pm 54$ & $50 \pm 48$ & $1.6 \pm 0.72$ & $0(0.0)$ \\
\hline $\begin{array}{l}\text { Xylinas } \\
\text { et al. (32) }\end{array}$ & $\begin{array}{l}\text { CHU Henri Mondor, } \\
\text { Créteil, France }\end{array}$ & RS & 12 & $6(50.0)$ & NA & 144 [120-180] & 60 [20-200] & $3.4[\mathrm{NA}]$ & $0(0.0)$ \\
\hline $\begin{array}{l}\text { Ploumidis } \\
\text { et al. (33) }\end{array}$ & $\begin{array}{l}\text { OLV Vattikuti Robotic } \\
\text { Surgery Institute, Aalst, } \\
\text { Belgium }\end{array}$ & RS & 95 & NA & NA & 101 [90-120] & 30 [20-50] & 4 [3-5] & $0(0.0)$ \\
\hline
\end{tabular}

Table 1 (continued) 
Table 1 (continued)

\begin{tabular}{|c|c|c|c|c|c|c|c|c|c|}
\hline Study & Institution & $\begin{array}{l}\text { Study } \\
\text { design }\end{array}$ & $\begin{array}{l}\text { Robotic } \\
\text { cases }\end{array}$ & $\begin{array}{l}\text { Concomitant } \\
\text { anti-incontinence } \\
\text { procedure } \\
\text { rate }(\%)\end{array}$ & $\begin{array}{l}\text { Concomitant } \\
\text { hysterectomy } \\
\text { rate }(\%)\end{array}$ & $\begin{array}{l}\text { Median/mean } \\
\text { operative time, } \\
\text { min }\end{array}$ & $\begin{array}{l}\text { Median/ } \\
\text { mean blood } \\
\text { loss, mL }\end{array}$ & $\begin{array}{l}\text { In-hospital } \\
\text { stay, d }\end{array}$ & $\begin{array}{l}\text { Transfusion } \\
\text { rate }(\%)\end{array}$ \\
\hline $\begin{array}{l}\text { Elliott } \\
\text { et al. (34) }\end{array}$ & $\begin{array}{l}\text { Stanford University, } \\
\text { Stanford, CA, USA }\end{array}$ & RS & 40 & NA & NA & 226 [NA] & NA & $1[0-10]$ & $0(0.0)$ \\
\hline $\begin{array}{l}\text { Anger } \\
\text { et al. (36) }\end{array}$ & $\begin{array}{l}\text { Cedars-Sinai Medical } \\
\text { Center, Los Angeles, CA, } \\
\text { USA }\end{array}$ & RCT & 40 & $26(65.0)$ & $25(62.5)$ & $202.8 \pm 46.0$ & $41.3 \pm 37.0$ & NA & NA \\
\hline $\begin{array}{l}\text { Barboglio } \\
\text { et al. (37) }\end{array}$ & $\begin{array}{l}\text { Dartmouth-Hitchcock } \\
\text { Medical Center, Lebanon, } \\
\mathrm{NH}, \text { USA }\end{array}$ & RS & 127 & NA & NA & NA & NA & NA & $1(0.7)$ \\
\hline $\begin{array}{l}\text { Geller } \\
\text { et al. (39) }\end{array}$ & $\begin{array}{l}\text { University of North } \\
\text { Carolina at Chapel Hill, } \\
\text { Chapel Hill, NC, USA }\end{array}$ & RS & 23 & NA & NA & NA & $151 \pm 111$ & NA & NA \\
\hline $\begin{array}{l}\text { Tan-Kim } \\
\text { et al. (40) }\end{array}$ & $\begin{array}{l}\text { University of California, } \\
\text { San Diego, CA, USA }\end{array}$ & RS & 43 & NA & NA & $281 \pm 58$ & $86 \pm 42$ & $1 \pm 0$ & $1(2.3)$ \\
\hline $\begin{array}{l}\text { Louis- } \\
\text { Sylvestre } \\
\text { and Herry } \\
\text { (41) }\end{array}$ & $\begin{array}{l}\text { Institut Mutualiste } \\
\text { Montsouris, Paris, } \\
\text { France }\end{array}$ & RS & 90 & NA & $49(54.4)$ & 246 [180-415] & NA & 3.48 [2-11] & $0(0.0)$ \\
\hline $\begin{array}{l}\text { Akl } \\
\text { et al. (44) }\end{array}$ & $\begin{array}{l}\text { Mayo Clinic, Phoenix, } \\
\text { AZ, USA }\end{array}$ & RS & 80 & NA & $4(5.0)$ & $197.9 \pm 66.8$ & $\begin{array}{l}96.8 \\
{[25-3,000]}\end{array}$ & $2.6[\mathrm{NA}]$ & $0(0.0)$ \\
\hline $\begin{array}{l}\text { Antosh } \\
\text { et al. (45) }\end{array}$ & $\begin{array}{l}\text { Washington Hospital } \\
\text { Center, Washington, DC, } \\
\text { USA }\end{array}$ & RS & 65 & $20(30.7)$ & $28(43.0)$ & 334 [205-537] & $\begin{array}{l}50 \\
{[10-1,000]}\end{array}$ & $1[1-5]$ & $0(0.0)$ \\
\hline $\begin{array}{l}\text { Matanes } \\
\text { et al. (46) }\end{array}$ & $\begin{array}{l}\text { Rambam Health Care } \\
\text { Campus, and Ruth and } \\
\text { Bruce Rappaport Faculty } \\
\text { of Medicine, Technion, } \\
\text { Haifa, Israel }\end{array}$ & RS & 25 & $2(8.0)$ & $20(80.0)$ & 190 [114-308] & 30 [10-300] & $2[1-4]$ & $0(0.0)$ \\
\hline $\begin{array}{l}\text { Salamon } \\
\text { and Culligan } \\
(47)\end{array}$ & $\begin{array}{l}\text { Division of } \\
\text { Urogynecology, } \\
\text { Atlantic Health System, } \\
\text { Morristown, NJ, USA }\end{array}$ & RS & 64 & 38 (59.3) & $41(64.0)$ & $165.6 \pm 23.0$ & $58.1 \pm 55.9$ & $1[1-2]$ & $0(0.0)$ \\
\hline
\end{tabular}

Table 1 (continued) 
Table 1 (continued)

\begin{tabular}{|c|c|c|c|c|c|c|c|c|c|}
\hline Study & Institution & $\begin{array}{l}\text { Study } \\
\text { design }\end{array}$ & $\begin{array}{l}\text { Robotic } \\
\text { cases }\end{array}$ & $\begin{array}{l}\text { Concomitant } \\
\text { anti-incontinence } \\
\text { procedure } \\
\text { rate }(\%)\end{array}$ & $\begin{array}{l}\text { Concomitant } \\
\text { hysterectomy } \\
\text { rate }(\%)\end{array}$ & $\begin{array}{l}\text { Median/mean } \\
\text { operative time, } \\
\text { min }\end{array}$ & $\begin{array}{l}\text { Median/ } \\
\text { mean blood } \\
\text { loss, mL }\end{array}$ & $\begin{array}{l}\text { In-hospital } \\
\text { stay, d }\end{array}$ & $\begin{array}{l}\text { Transfusion } \\
\text { rate (\%) }\end{array}$ \\
\hline $\begin{array}{l}\text { Culligan } \\
\text { et al. (48) }\end{array}$ & $\begin{array}{l}\text { Atlantic Health System, } \\
\text { Morristown, NJ, USA }\end{array}$ & PS & 150 & $128(85.3)$ & NA & $148 \pm 27.6$ & $51.2 \pm 32$ & $1[\mathrm{NA}]$ & $0(0.0)$ \\
\hline $\begin{array}{l}\text { Siddiqui } \\
\text { et al. (50) }\end{array}$ & $\begin{array}{l}\text { Duke University Medical } \\
\text { Center, Durham, NC, } \\
\text { USA }\end{array}$ & $\mathrm{RS}$ & 125 & $52(41.6)$ & $61(48.8)$ & NA & $90 \pm 89.3$ & NA & $1(0.8)$ \\
\hline $\begin{array}{l}\text { Bedaiwy } \\
\text { et al. (51) }\end{array}$ & $\begin{array}{l}\text { University Hospitals } \\
\text { Case Medical Center, } \\
\text { Cleveland, OH, USA }\end{array}$ & $\mathrm{RS}$ & 41 & $27(65.8)$ & $27(65.8)$ & $328.5 \pm 56$ & $50 \pm 50$ & NA & $1(2.4)$ \\
\hline Overall & - & - & 2,916 & $729(25.0)$ & $634(21.7)$ & 226 [90-404] & $56[5-1,500]$ & $1.55[1-16]$ & $5(0.1)$ \\
\hline
\end{tabular}

RSC, robotic sacrocolpopexy; PS, prospective study; RCT, randomized controlled trial; RS, retrospective study; NA, not applicable.

\section{Surgical-related complications}

A summary of surgical-related complications in the RSC series is presented in Tables 2,3. Intraoperative complications and postoperative complications occurred in 74 and 360 patients out of the 2,768 RSC series, respectively, and 58 cases of mesh erosion were included in the postoperative complications. We classified the intraoperative complications into three grades of severity according to the Satava severity system: grade 1 complications, no consequence for the patient; grade 2 complications, treated intraoperatively with endoscopic surgery or required endoscopic retreatment; and grade 3 complications, incidents requiring open or laparoscopic surgery $(37,55)$. The severity of postoperative complications was classified using the Clavien-Dindo severity system, ranging from a slight deviation from the normal postoperative course (grade 1) to death (grade 5) (56).

Procedural complications were observed in $2.7 \%$ $(n=74)$ of cases. There were $0.07 \%(n=2), 1.84 \%(n=51)$, and $0.14 \%(n=4)$ complications classified as grade 1,2 , and 3, respectively. Bladder injury [48.6\% $(n=37)]$ was the most common intraoperative complication, and intestinal injury, vascular injury, ureteral injury, and others were $11.8 \%(\mathrm{n}=9), 10.5 \%(\mathrm{n}=8), 3.94 \%(\mathrm{n}=3)$, and $25 \%(\mathrm{n}=19)$, respectively. Although the occurrence rate of intraoperative complications was low, postoperative complications were reported at a rate of $13.0 \%(360 / 2,768)$. The numbers of postoperative grade $1,2,3 \mathrm{a}, 3 \mathrm{~b}$, and $4 \mathrm{a}$ complications were 73 (2.63\%), 85 (3.07\%), $27(0.97 \%), 31(1.11 \%)$, and 5 $(0.18 \%)$, respectively, with no grade $4 \mathrm{~b}$ and 5 complications recorded. In the postoperative grade 4a complication, one patient developed postoperative surgical emphysema and pulmonary edema, and she was readmitted to the surgical intensive care unit (SICU) (50). Another patient had a postoperative acute myocardial infarction, which was successfully treated (17). As the predominant long-term postoperative complication, mesh erosion occurred at a rate of $2.09 \%(n=58)$. Urinary dysfunction [40\% ( $n=144)$ ] was the most of the postoperative complications, including urinary infection, de novo urinary stress incontinence, and dysuria. The prevalence of ileus and defecatory dysfunction, wound infection/abscess, dyspareunia, and pelvic hematoma were $13.0 \%(n=47), 5.55 \%(n=20), 3.61 \%$ $(n=13)$, and $1.11 \%(n=4)$, respectively. The predominance of other postoperative complications, such as vaginal bleeding, vaginal cuff dehiscence pelvic pain, pneumonia, deep venous thrombosis, and vaginal cuff dehiscence, was $20.6 \%(n=79)$.

A total of 40 patients had been converted from a robot-assisted approach to other approaches, of which 6 
Table $2 \mathrm{~A}$ summary of intraoperative complications in the RSC series

\begin{tabular}{|c|c|c|c|c|c|c|c|c|c|c|c|c|c|}
\hline \multirow{2}{*}{ Study } & \multirow{2}{*}{ Institution } & \multirow{2}{*}{ Study design } & \multirow{2}{*}{ obotic cases } & \multicolumn{6}{|c|}{ Intraoperative complications (\%) } & \multirow{2}{*}{ Conversion (\%) } & \multicolumn{3}{|c|}{ Savata classification (\%) } \\
\hline & & & & Bladder injury & Bowel injury & Vascular injury & Ureteral injury & Other injury & Total & & Grade 1 & Grade 2 & Grade 3 \\
\hline Ferrando et al. (6) & Cleveland Clinic, Cleveland, OH, USA & RCT & 24 & $0(0.0)$ & $0(0.0)$ & $0(0.0)$ & $0(0.0)$ & $0(0.0)$ & $0(0.0)$ & $0(0.0)$ & $0(0.0)$ & $0(0.0)$ & $0(0.0)$ \\
\hline Geller et al. (7) & University of North Carolina at Chapel Hill, Chapel Hill, NC, USA & RS & 147 & NA & NA & NA & NA & NA & NA & NA & NA & NA & NA \\
\hline Elliott et al. (8) & Mayo Clinic, Rochester, MN, USA & PS & 42 & NA & NA & NA & NA & NA & NA & $2(4.76)$ & NA & NA & NA \\
\hline Thubert et al. (53) & Pitié Salpétrière Hospital, APHP, Paris, France & RCT & 95 & $3(100.0)$ & $0(0.0)$ & $0(0.0)$ & $0(0.0)$ & $0(0.0)$ & $3(3.15)$ & $1(1.05)$ & $0(0.0)$ & $3(3.15)$ & $0(0.0)$ \\
\hline Mueller et al. (9) & Loyola University Chicago Stritch School of Medicine, Chicago, IL, USA & RCT & 40 & NA & NA & NA & NA & NA & NA & NA & NA & NA & NA \\
\hline Shariati et al. (11) & UMDNJ New Jersey Medical School, Newark, NJ, USA & RS & 77 & $0(0.0)$ & 1 (16.6) & $0(0.0)$ & $0(0.0)$ & $5(83.3)$ & $6(7.79)$ & $1(1.29)$ & $0(0.0)$ & $5(6.49)$ & $1(1.29)$ \\
\hline Elliott et al. (13) & Mayo Clinic, Rochester, MN, USA & PS & 20 & NA & NA & NA & NA & NA & NA & $1(5.0)$ & NA & NA & NA \\
\hline Paraiso et al. (15) & Cleveland Clinic, Cleveland, OH, USA & RCT & 40 & $2(40.0)$ & $1(20.0)$ & $0(0.0)$ & $0(0.0)$ & $2(40.0)$ & $5(12.5)$ & $3(7.5)$ & $1(2.5)$ & $3(7.5)$ & $1(2.5)$ \\
\hline Chan et al. (16) & $\begin{array}{l}\text { The Chinese University of Hong Kong, Prince of Wales Hospital, Hong } \\
\text { Kong, China }\end{array}$ & RS & 16 & $2(66.6)$ & $0(0.0)$ & $0(0.0)$ & 1 (33.3) & $0(0.0)$ & $3(18.7)$ & NA & $0(0.0)$ & $2(12.5)$ & $1(6.25)$ \\
\hline Moreno Sierra et al. (17) & Hospital Clinico San Carlos, Universidad Complutense, Madrid, Spain & PS & 31 & $1(50.0)$ & $0(0.0)$ & $0(0.0)$ & $0(0.0)$ & $1(50.0)$ & $2(6.45)$ & $1(3.22)$ & $0(0.0)$ & $2(6.45)$ & $0(0.0)$ \\
\hline Shimko et al. (18) & Mayo Clinic, Rochester, MN, USA & RS & 40 & NA & NA & NA & NA & NA & NA & $3(7.5)$ & NA & $2(5.0)$ & NA \\
\hline Linder et al. (19) & Mayo Clinic, Rochester, MN, USA & PS & 84 & NA & NA & NA & NA & NA & NA & $14(16.6)$ & NA & NA & NA \\
\hline Elliott et al. (20) & Mayo Clinic, Rochester, MN, USA & PS & 30 & NA & NA & NA & NA & NA & NA & $1(3.33)$ & NA & NA & NA \\
\hline Osmundsen et al. (21) & Oregon Health \& Science University, Portland, OR, USA & RS & 102 & NA & NA & NA & NA & NA & NA & NA & NA & NA & NA \\
\hline Belsante et al. (22) & UT Southwestern Medical Centre, TX, USA & RS & 35 & NA & NA & NA & NA & NA & NA & NA & NA & NA & NA \\
\hline Jambusaria et al. (24) & Abington Memorial Hospital, Abington, PA, USA & RS & 38 & $1(100.0)$ & $0(0.0)$ & $0(0.0)$ & $0(0.0)$ & $0(0.0)$ & $1(2.63)$ & $1(2.63)$ & $0(0.0)$ & $1(2.63)$ & $0(0.0)$ \\
\hline Mueller et al. (26) & $\begin{array}{l}\text { The Loyola University Chicago, Stritch School of Medicine, Maywood, } \\
\text { IL,USA }\end{array}$ & RS & 226 & $3(100.0)$ & $0(0.0)$ & $0(0.0)$ & $0(0.0)$ & $0(0.0)$ & $3(1.32)$ & $0(0.0)$ & NA & NA & NA \\
\hline Nosti et al. (27) & $\begin{array}{l}\text { FPMRS Medstar Washington Hospital Center, Georgetown University } \\
\text { School of Medicine, Washington, DC, USA }\end{array}$ & RS & 262 & $4(80.0)$ & $0(0.0)$ & $1(20.0)$ & $0(0.0)$ & $0(0.0)$ & $5(1.90)$ & $1(0.38)$ & NA & NA & NA \\
\hline Borahay et al. (54) & $\begin{array}{l}\text { The University of Texas Medical Branch at Galveston, Galveston, TX, } \\
\text { USA }\end{array}$ & RS & 20 & NA & NA & NA & NA & $3(100.0)$ & $3(15.0)$ & $0(0.0)$ & $1(5.0)$ & $2(10.0)$ & $0(0.0)$ \\
\hline Geller et al. (28) & University of North Carolina at Chapel Hill, Chapel Hill, NC, USA & PS & 28 & NA & NA & NA & NA & NA & NA & NA & NA & NA & NA \\
\hline Unger et al. (29) & Cleveland Clinic, Cleveland, OH, USA & RS & 121 & $4(50.0)$ & $3(37.5)$ & 1 (12.5) & $0(0.0)$ & $0(0.0)$ & $8(6.61)$ & $5(4.13)$ & $0(0.0)$ & $5(4.13)$ & $0(0.0)$ \\
\hline Biler et al. (30) & $\begin{array}{l}\text { University of Health Sciences Tepecik Training and Research Hospital, } \\
\text { Izmir, Turkey }\end{array}$ & PS & 20 & $2(100.0)$ & $0(0.0)$ & $0(0.0)$ & $0(0.0)$ & $0(0.0)$ & $2(10.0)$ & $1(5.0)$ & NA & NA & NA \\
\hline Matthews et al. (31) & Virginia Commonwealth University Medical Center, Richmond, VA, USA & PS & 85 & $2(66.6)$ & $0(0.0)$ & $0(0.0)$ & $0(0.0)$ & $0(0.0)$ & $3(3.52)$ & $0(0.0)$ & $0(0.0)$ & $6(7.05)$ & $0(0.0)$ \\
\hline Ploumidis et al. (33) & OLV Vattikuti Robotic Surgery Institute, Aalst, Belgium & RS & 95 & $2(66.6)$ & $0(0.0)$ & $0(0.0)$ & $0(0.0)$ & $1(33.3)$ & $3(3.15)$ & NA & $0(0.0)$ & $3(3.15)$ & $0(0.0)$ \\
\hline Elliott et al. (34) & Stanford University, Stanford, CA, USA & RS & 40 & NA & NA & NA & NA & NA & NA & NA & NA & NA & NA \\
\hline Illiano et al. (35) & Andrology and Urogynecology Clinic, Santa Maria Terni, Italy & PS & 49 & NA & NA & NA & NA & NA & NA & NA & NA & NA & NA \\
\hline Anger et al. (36) & Cedars-Sinai Medical Center, Los Angeles, CA, USA & RCT & 40 & $0(0.0)$ & $1(50.0)$ & $1(50.0)$ & $0(0.0)$ & $0(0.0)$ & $2(5.0)$ & NA & $0(0.0)$ & $1(2.5)$ & $1(2.5)$ \\
\hline Barboglio et al. (37) & Dartmouth-Hitchcock Medical Center, Lebanon, NH, USA & RS & 127 & $0(0.0)$ & $2(100.0)$ & $0(0.0)$ & $0(0.0)$ & $0(0.0)$ & $2(1.57)$ & NA & $0(0.0)$ & $2(1.57)$ & $0(0.0)$ \\
\hline
\end{tabular}




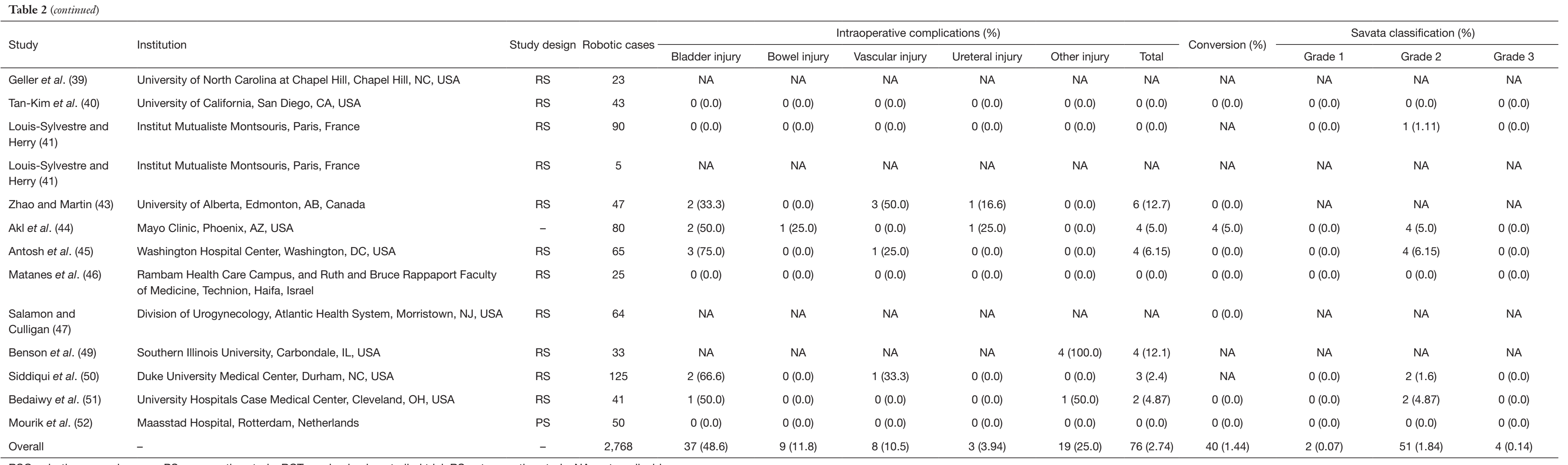




\begin{tabular}{|c|c|c|c|c|c|c|c|c|c|c|c|c|c|c|c|c|c|}
\hline \multirow[b]{2}{*}{ Study } & \multirow[b]{2}{*}{ Institution } & \multirow[b]{2}{*}{ Study design } & \multirow[b]{2}{*}{ Robotic cases } & \multicolumn{7}{|c|}{ Postoperative complication (\%) } & \multicolumn{7}{|c|}{ Clavien-Dindo classification (\%) } \\
\hline & & & & Pelvic hematoma & Wound infection & $\begin{array}{l}\text { Urinary } " I \\
\text { dysfunction }\end{array}$ & $\begin{array}{l}\text { lleus and defecatory } \\
\text { dysfunction }\end{array}$ & Dyspareunia & Mesh erosion & Total & Grade 1 & Grade 2 & Grade 3a & Grade $3 b$ & Grade 4a & Grade $4 \mathrm{~b}$ & Grade 5 \\
\hline Ferrandom et al. (6) & Cleveland Clinic, Cleveland, OH, USA & $\mathrm{RCT}$ & 24 & $0(0.0)$ & $0(0.0)$ & $0(0.0)$ & $0(0.0)$ & $0(0.0)$ & $0(0.0)$ & $0(0.0)$ & $0(0.0)$ & $0(0.0)$ & $0(0.0)$ & $0(0.0)$ & $0(0.0)$ & $0(0.0)$ & $0(0.0)$ \\
\hline Geller et al. (7) & University of North Carolina at Chapel Hill, Chapel Hill, NC, USA & RS & 147 & NA & NA & NA & NA & NA & NA & NA & NA & NA & NA & NA & NA & NA & NA \\
\hline Thubert et al. (53) & Pitié Salpétrière Hospital, APHP, Paris, France & RCT & 95 & $0(0.0)$ & $0(0.0)$ & $21(80.7)$ & $3(11.5)$ & $0(0.0)$ & $2(2.10)$ & $26(27.3)$ & NA & NA & NA & NA & NA & NA & NA \\
\hline Mueller et al. (9) & Loyola University Chicago Stritch School of Medicine, Chicago, IL, USA & RCT & 40 & NA & NA & NA & NA & NA & NA & NA & NA & NA & NA & NA & NA & NA & NA \\
\hline Shariati et al. (11) & UMDNJ New Jersey Medical School, Newark, NJ, USA & RS & 77 & $0(0.0)$ & $0(0.0)$ & $5(29.4)$ & $5(29.4)$ & $5(29.4)$ & $0(0.0)$ & $17(22.0)$ & $5(6.49)$ & $9(11.6)$ & $3(3.89)$ & $0(0.0)$ & $0(0.0)$ & $0(0.0)$ & $0(0.0)$ \\
\hline Elliott et al. (13) & Mayo Clinic, Rochester, MN, USA & PS & 20 & $0(0.0)$ & $2(50.0)$ & $0(0.0)$ & $0(0.0)$ & $0(0.0)$ & $1(5.0)$ & $4(20.0)$ & $0(0.0)$ & $2(10.0)$ & $1(5.0)$ & $0(0.0)$ & $0(0.0)$ & $0(0.0)$ & $0(0.0)$ \\
\hline Awad et al. (14) & Ruth and Bruce Rappaport Faculty of Medicine, Technion, Haifa, Israel & RS & 40 & $1(25.0)$ & $0(0.0)$ & $3(75.0)$ & $0(0.0)$ & $0(0.0)$ & NA & $4(10.0)$ & NA & NA & NA & NA & NA & NA & NA \\
\hline Paraiso et al. (15) & Cleveland Clinic, Cleveland, OH, USA & RCT & 40 & $0(0.0)$ & $3(20.0)$ & $5(33.3)$ & $2(13.3)$ & $0(0.0)$ & $2(5.0)$ & $15(37.5)$ & $0(0.0)$ & $0(0.0)$ & $10(25.0)$ & $0(0.0)$ & $3(7.5)$ & $0(0.0)$ & $0(0.0)$ \\
\hline Chan et al. (16) & $\begin{array}{l}\text { The Chinese University of Hong Kong, Prince of Wales Hospital, Hong } \\
\text { Kong }\end{array}$ & RS & 16 & NA & NA & NA & NA & NA & NA & $2(12.5)$ & $(0.0)$ & $1(6.25)$ & $(0.0)$ & $1(6.25)$ & $0(0.0)$ & $0(0.0)$ & $0(0.0)$ \\
\hline $\begin{array}{l}\text { Moreno Sierra } \\
\text { et al. (17) }\end{array}$ & Hospital Clinico San Carlos, Universidad Complutense, Madrid, Spain & PS & 31 & $0(0.0)$ & $0(0.0)$ & $0(0.0)$ & $0(0.0)$ & $0(0.0)$ & $0(0.0)$ & $2(6.45)$ & $0(0.0)$ & $0(0.0)$ & $0(0.0)$ & $1(3.22)$ & $1(3.22)$ & $0(0.0)$ & $0(0.0)$ \\
\hline Shimko et al. (18) & Mayo Clinic, Rochester, MN, USA & RS & 40 & $0(0.0)$ & $0(0.0)$ & $9(81.8)$ & $0(0.0)$ & $0(0.0)$ & $2(5.0)$ & $11(27.5)$ & $1(2.5)$ & $1(2.5)$ & $(0.0)$ & $2(5.0)$ & $0(0.0)$ & $0(0.0)$ & $0(0.0)$ \\
\hline Linder et al. (19) & Mayo Clinic, Rochester, MN, USA & PS & 84 & NA & NA & NA & NA & NA & NA & NA & $0(0.0)$ & $0(0.0)$ & $0(0.0)$ & $2(2.38)$ & $0(0.0)$ & $0(0.0)$ & $0(0.0)$ \\
\hline Elliott et al. (20) & Mayo Clinic, Rochester, MN, USA & PS & 30 & $0(0.0)$ & $2(66.6)$ & $0(0.0)$ & $0(0.0)$ & $0(0.0)$ & $0(0.0)$ & $3(10.0)$ & $1(3.33)$ & $2(6.66)$ & $0(0.0)$ & $0(0.0)$ & $0(0.0)$ & $0(0.0)$ & $0(0.0)$ \\
\hline $\begin{array}{l}\text { Osmundsen } \\
\text { et al. (21) }\end{array}$ & Oregon Health \& Science University, Portland, OR, USA & RS & 102 & NA & NA & NA & NA & NA & $8(7.84)$ & NA & NA & NA & NA & NA & NA & NA & NA \\
\hline Belsante et al. (22) & UT Southwestern Medical Centre, TX, USA & RS & 35 & NA & NA & NA & NA & NA & NA & NA & NA & NA & NA & NA & NA & NA & NA \\
\hline Pulliam et al. (23) & $\begin{array}{l}\text { Massachusetts General Hospital, Harvard Medical School, Boston, MA, } \\
\text { USA }\end{array}$ & RS & 43 & NA & NA & NA & NA & NA & NA & $1(2.32)$ & $0(0.0)$ & $1(2.32)$ & $0(0.0)$ & $0(0.0)$ & $0(0.0)$ & $0(0.0)$ & $0(0.0)$ \\
\hline $\begin{array}{l}\text { Jambusaria } \\
\text { et al. (24) }\end{array}$ & Abington Memorial Hospital, Abington, PA, USA & RS & 38 & $0(0.0)$ & $0(0.0)$ & $0(0.0)$ & $0(0.0)$ & $0(0.0)$ & $1(2.63)$ & $2(5.26)$ & $0(0.0)$ & $0(0.0)$ & $0(0.0)$ & $1(2.63)$ & $0(0.0)$ & $0(0.0)$ & $0(0.0)$ \\
\hline Mueller et al. (26) & $\begin{array}{l}\text { The Loyola University Chicago, Stritch School of Medicine, Maywood, } \\
\text { IL,USA }\end{array}$ & RS & 226 & $0(0.0)$ & $0(0.0)$ & $0(0.0)$ & $8(80.0)$ & NA & $2(0.88)$ & $10(4.42)$ & $0(0.0)$ & $5(2.21)$ & $0(0.0)$ & $3(1.32)$ & $0(0.0)$ & $0(0.0)$ & $0(0.0)$ \\
\hline Nosti et al. (27) & $\begin{array}{l}\text { FPMRS Medstar Washington Hospital Center, Georgetown University } \\
\text { School of Medicine, Washington, DC, USA }\end{array}$ & RS & 262 & NA & NA & NA & NA & NA & $5(1.90)$ & $5(1.90)$ & NA & NA & NA & NA & NA & NA & NA \\
\hline Borahay et al. (54) & The University of Texas Medical Branch, Galveston, Texas, USA & PS & 20 & NA & NA & NA & NA & NA & $0(0.0)$ & $3(15.0)$ & $2(10.0)$ & $0(0.0)$ & $0(0.0)$ & $1(5.0)$ & $0(0.0)$ & $0(0.0)$ & $0(0.0)$ \\
\hline Geller et al. (28) & University of North Carolina at Chapel Hill, Chapel Hill, NC, USA & PS & 28 & NA & NA & NA & NA & NA & $2(7.14)$ & $4(14.2)$ & $0(0.0)$ & $2(7.14)$ & $0(0.0)$ & $0(0.0)$ & $0(0.0)$ & $0(0.0)$ & $0(0.0)$ \\
\hline Unger et al. (29) & Cleveland Clinic, Cleveland, OH, USA & RS & 121 & $0(0.0)$ & $1(7.14)$ & $0(0.0)$ & $0(0.0)$ & $0(0.0)$ & $4(3.30)$ & $14(11.5)$ & $5(4.13)$ & $5(4.13)$ & $0(0.0)$ & $0(0.0)$ & $0(0.0)$ & $0(0.0)$ & $0(0.0)$ \\
\hline Biler et al. (30) & $\begin{array}{l}\text { University of Health Sciences Tepecik Training and Research Hospital, } \\
\text { Izmir, Turkey }\end{array}$ & PS & 20 & $0(0.0)$ & $0(0.0)$ & $1(16.6)$ & 1 (16.6) & $0(0.0)$ & $0(0.0)$ & $6(30.0)$ & $4(20.0)$ & $1(5.0)$ & $0(0.0)$ & $1(5.0)$ & $0(0.0)$ & $0(0.0)$ & $0(0.0)$ \\
\hline Matthews et al. (31) & Virginia Commonwealth University Medical Center, Richmond, VA, USA & PS & 85 & $0(0.0)$ & $0(0.0)$ & $11(57.8)$ & $6(31.5)$ & $0(0.0)$ & $1(1.17)$ & $19(22.3)$ & $1(1.17)$ & $15(17.6)$ & $0(0.0)$ & $2(2.35)$ & $0(0.0)$ & $0(0.0)$ & $0(0.0)$ \\
\hline Ploumidis et al. (33) & OLV Vattikuti Robotic Surgery Institute, Aalst, Belgium & RS & 95 & $0(0.0)$ & $0(0.0)$ & $10(90.9)$ & $0(0.0)$ & $0(0.0)$ & $1(1.05)$ & $11(11.5)$ & $1(1.05)$ & $1(1.05)$ & $0(0.0)$ & $1(1.05)$ & $0(0.0)$ & $0(0.0)$ & $0(0.0)$ \\
\hline Elliott et al. (34) & Stanford University, Stanford, CA, USA & RS & 40 & NA & NA & NA & NA & NA & NA & NA & NA & NA & NA & NA & NA & NA & NA \\
\hline Illiano et al. (35) & Andrology and Urogynecology Clinic, Santa Maria Terni, Italy & PS & 49 & $1(5.26)$ & $0(0.0)$ & $6(31.5)$ & $6(31.5)$ & $4(21.0)$ & $2(4.08)$ & $19(38.7)$ & $9(18.3)$ & $1(2.04)$ & $0(0.0)$ & $0(0.0)$ & $0(0.0)$ & $0(0.0)$ & $0(0.0)$ \\
\hline
\end{tabular}

Table 3 (continued) 
Geller et al. (39) University of North Carolina at Chapel Hill, Chapel Hill, NC, USA

Tan-Kim et al. (40) University of California, San Diego, CA, USA

Louis-Sylvestre and Institut Mutualiste Montsouris, Paris, France

Herry (41)

Di Marco et al. (42) Mayo Clinic, Rochester, MN, USA

Zhao and Martin (43) University of Alberta, Edmonton, AB, Canada

Akl et al. (44) Mayo Clinic, Phoenix, AZ, USA

Antosh et al. (45) Washington Hospital Center, Washington, DC, USA

Matanes et al. (46) Rambam Health Care Campus, and Ruth and Bruce Rappaport Faculty of Medicine, Technion, Haifa, Israe

Division of Urogynecology, Atlantic Health System, Morristown, NJ, USA

Culligan (47)

Benson et al. (49) Southern Illinois University, Carbondale, IL, USA

Siddiqui et al. (50) Duke University Medical Center, Durham, NC, USA

Bedaiwy et al. (51) University Hospitals Case Medical Center, Cleveland, OH, USA

Mourik et al. (52) Maasstad Hospital, Rotterdam, Netherlands

\begin{tabular}{|c|c|c|c|c|c|c|c|c|c|c|c|c|c|c|c|}
\hline $\mathrm{RCT}$ & 40 & NA & NA & NA & NA & NA & NA & NA & NA & NA & NA & NA & NA & NA & NA \\
\hline RS & 127 & $0(0.0)$ & $0(0.0)$ & $20(76.9)$ & $3(11.5)$ & $0(0.0)$ & $3(2.36)$ & $26(20.4)$ & $2(1.57)$ & $13(10.2)$ & $0(0.0)$ & $7(5.51)$ & $0(0.0)$ & $0(0.0)$ & $0(0.0)$ \\
\hline RCT & 20 & $0(0.0)$ & $0(0.0)$ & $0(0.0)$ & $0(0.0)$ & $0(0.0)$ & $0(0.0)$ & $0(0.0)$ & $0(0.0)$ & $0(0.0)$ & $0(0.0)$ & $0(0.0)$ & $0(0.0)$ & $0(0.0)$ & $0(0.0)$ \\
\hline RS & 23 & NA & NA & NA & NA & NA & $2(8.69)$ & $4(17.3)$ & $0(0.0)$ & $2(8.69)$ & $0(0.0)$ & $0(0.0)$ & $0(0.0)$ & $0(0.0)$ & $0(0.0)$ \\
\hline PS & 43 & NA & NA & NA & NA & NA & $2(4.65)$ & $5(11.6)$ & $1(2.32)$ & $2(4.65)$ & $0(0.0)$ & $0(0.0)$ & $0(0.0)$ & $0(0.0)$ & $0(0.0)$ \\
\hline PS & 90 & $0(0.0)$ & $0(0.0)$ & $0(0.0)$ & 1 (12.5) & $4(50.0)$ & $1(1.11)$ & $8(8.88)$ & $0(0.0)$ & $0(0.0)$ & $0(0.0)$ & $0(0.0)$ & $0(0.0)$ & $0(0.0)$ & $0(0.0)$ \\
\hline PS & 5 & NA & NA & NA & NA & NA & NA & $1(20.0)$ & $1(20.0)$ & $0(0.0)$ & $0(0.0)$ & $0(0.0)$ & $0(0.0)$ & $0(0.0)$ & $0(0.0)$ \\
\hline RS & 47 & $2(5.55)$ & $1(2.77)$ & $16(44.4)$ & $2(5.55)$ & $0(0.0)$ & $1(2.12)$ & $36(76.5)$ & $25(53.1)$ & $7(14.8)$ & $2(4.25)$ & $2(4.25)$ & $0(0.0)$ & $0(0.0)$ & $0(0.0)$ \\
\hline PS & 80 & $0(0.0)$ & $1(9.09)$ & $0(0.0)$ & $1(9.09)$ & $(0.0)$ & $5(6.25)$ & 11 (13.7) & $0(0.0)$ & $2(2.5)$ & $4(5.0)$ & $0(0.0)$ & $0(0.0)$ & $0(0.0)$ & $0(0.0)$ \\
\hline RS & 65 & NA & $2(7.69)$ & $19(73.0)$ & NA & NA & $2(3.07)$ & $26(40.0)$ & $0(0.0)$ & $3(4.61)$ & $0(0.0)$ & $2(3.07)$ & $0(0.0)$ & $0(0.0)$ & $0(0.0)$ \\
\hline RS & 25 & $0(0.0)$ & $0(0.0)$ & $0(0.0)$ & $0(0.0)$ & $0(0.0)$ & NA & $1(4.0)$ & $0(0.0)$ & $0(0.0)$ & $0(0.0)$ & $1(4.0)$ & $0(0.0)$ & $0(0.0)$ & $0(0.0)$ \\
\hline PS & 64 & NA & NA & NA & NA & NA & $1(1.56)$ & $3(4.68)$ & $0(0.0)$ & $0(0.0)$ & $1(1.56)$ & $1(1.56)$ & $0(0.0)$ & $0(0.0)$ & $0(0.0)$ \\
\hline RS & 33 & NA & NA & NA & NA & NA & $0(0.0)$ & $4(12.1)$ & $0(0.0)$ & $2(6.06)$ & $0(0.0)$ & $2(6.06)$ & $0(0.0)$ & $0(0.0)$ & $0(0.0)$ \\
\hline RS & 125 & $0(0.0)$ & $6(27.2)$ & $0(0.0)$ & $7(31.8)$ & $0(0.0)$ & $3(2.4)$ & $22(17.6)$ & NA & NA & NA & NA & NA & NA & NA \\
\hline RS & 41 & $0(0.0)$ & $0(0.0)$ & $18(64.2)$ & $1(3.57)$ & $0(0.0)$ & $3(7.31)$ & 28 (68.2) & $15(36.5)$ & $6(14.6)$ & $3(7.31)$ & $1(2.43)$ & $1(2.43)$ & $0(0.0)$ & $0(0.0)$ \\
\hline PS & 50 & $0(0.0)$ & $0(0.0)$ & $0(0.0)$ & 1 (33.3) & $0(0.0)$ & $0(0.0)$ & $3(6.0)$ & $0(0.0)$ & $0(0.0)$ & $1(2.0)$ & $0(0.0)$ & $0(0.0)$ & $0(0.0)$ & $0(0.0)$ \\
\hline - & 2,768 & $4(1.11)$ & $20(5.55)$ & $144(40.0)$ & $47(13.0)$ & $13(3.61)$ & $58(2.09)$ & $\begin{array}{c}360 \\
(13.0)\end{array}$ & $73(2.63)$ & $85(3.07)$ & $27(0.97)$ & $31(1.11)$ & $5(0.18)$ & $0(0.0)$ & $0(0.0)$ \\
\hline
\end{tabular}

RSC, robotic sacrocolpopexy; PS, prospective study; RCT, randomized controlled trial; RS, retrospective study; NA, not applicable. 


\begin{tabular}{|c|c|c|c|c|c|c|c|c|c|c|c|c|c|c|c|}
\hline \multirow[b]{2}{*}{ Study } & \multirow[b]{2}{*}{ Institution } & \multirow[b]{2}{*}{ Study design } & \multirow[b]{2}{*}{ Robotic cases } & \multirow[b]{2}{*}{ Follow up cases } & \multirow[b]{2}{*}{$\begin{array}{l}\text { Median follow } \\
\text { up duration }\end{array}$} & \multicolumn{5}{|c|}{ Prolapse recurrence (\%) } & \multicolumn{5}{|c|}{ Reoperation for prolapse recurrence (\%) } \\
\hline & & & & & & Anterior & Apical & Posterior & $\begin{array}{l}\text { Anterior and } \\
\text { posterior }\end{array}$ & Total & $\begin{array}{l}\text { Vaginal colporrhaphy } \\
\text { or sacrocolpopexy }\end{array}$ & RSC & LSC & ASC & Total \\
\hline Ferrando et al. (6) & Cleveland Clinic, Cleveland, OH, USA & $\mathrm{RCT}$ & 24 & $24(100.0)$ & $6[\mathrm{NA}]$ & $0(0.0)$ & $0(0.0)$ & $0(0.0)$ & $0(0.0)$ & $0(0.0)$ & $0(0.0)$ & $0(0.0)$ & $0(0.0)$ & $0(0.0)$ & $0(0.0)$ \\
\hline Elliott et al. (8) & Mayo Clinic, Rochester, MN, USA & PS & 42 & $42(100.0)$ & 36 [12-48] & $0(0.0)$ & $1(50.0)$ & $1(50.0)$ & $0(0.0)$ & $2(4.76)$ & $1(100.0)$ & $0(0.0)$ & $0(0.0)$ & $0(0.0)$ & $1(50.0)$ \\
\hline Thubert et al. (53) & Pitié Salpétrière Hospital, APHP, Paris, France & $\mathrm{RCT}$ & 78 & $78(100.0)$ & $12[6-19.75]$ & $1(50.0)$ & $0(0.0)$ & $1(50.0)$ & $0(0.0)$ & $2(2.56)$ & $2(100.0)$ & $0(0.0)$ & $0(0.0)$ & $0(0.0)$ & $2(100.0)$ \\
\hline Paraiso et al. (11) & UMDNJ New Jersey Medical School, Newark, NJ, USA & RS & 77 & $53(68.8)$ & NA & NA & NA & NA & NA & $3(5.66)$ & NA & NA & NA & NA & NA \\
\hline Elliott et al. (13) & Mayo Clinic, Rochester, MN, USA & PS & 20 & $20(100.0)$ & $5.1[1-12]$ & $0(0.0)$ & $0(0.0)$ & $1(100.0)$ & $0(0.0)$ & $1(5.0)$ & NA & NA & NA & NA & NA \\
\hline Paraiso et al. (15) & Cleveland Clinic, Cleveland, OH, USA & $\mathrm{RCT}$ & 40 & $26(65.0)$ & $12[\mathrm{NA}]$ & NA & NA & NA & NA & $3(11.5)$ & NA & NA & NA & NA & NA \\
\hline Chan et al. (16) & The Chinese University of Hong Kong, Prince of Wales Hospital, Hong Kong, China & RS & 16 & $16(100.0)$ & $19[3-36]$ & NA & NA & NA & NA & $1(6.25)$ & $1(100.0)$ & $0(0.0)$ & $0(0.0)$ & $0(0.0)$ & $1(100.0)$ \\
\hline Moreno Sierra et al. (17) & Hospital Clinico San Carlos, Universidad Complutense Madrid, Spain & PS & 31 & $31(100.0)$ & $24.5[16-33]$ & $0(0.0)$ & $0(0.0)$ & $0(0.0)$ & $0(0.0)$ & $0(0.0)$ & $0(0.0)$ & $0(0.0)$ & $0(0.0)$ & $0(0.0)$ & $0(0.0)$ \\
\hline Shimko et al. (18) & Mayo Clinic, Rochester, MN, USA & RS & 40 & $40(100.0)$ & 62 [36-84] & $1(33.3)$ & $0(0.0)$ & $2(66.6)$ & $0(0.0)$ & $3(7.5)$ & NA & NA & NA & NA & NA \\
\hline Linder et al. (19) & Mayo Clinic, Rochester, MN, USA & PS & 84 & $70(83.3)$ & $72[39-114]$ & $2(50.0)$ & $1(25.0)$ & $1(25.0)$ & $0(0.0)$ & $4(5.71)$ & $3(75.0)$ & $0(0.0)$ & $0(0.0)$ & $1(25.0)$ & $4(100.0)$ \\
\hline Elliott et al. (20) & Mayo Clinic, Rochester, MN, USA & PS & 30 & $30(100.0)$ & 24 [16-39] & $0(0.0)$ & $1(50.0)$ & $1(50.0)$ & $0(0.0)$ & $2(6.66)$ & $1(50.0)$ & $0(0.0)$ & $0(0.0)$ & $1(50.0)$ & $2(100.0)$ \\
\hline Belsante et al. (22) & UT Southwestern Medical Centre, TX, USA & RS & 35 & $35(100.0)$ & $6[\mathrm{NA}]$ & $0(0.0)$ & $0(0.0)$ & $0(0.0)$ & $0(0.0)$ & $0(0.0)$ & $0(0.0)$ & $0(0.0)$ & $0(0.0)$ & $0(0.0)$ & $0(0.0)$ \\
\hline Kenton et al. (25) & Northwestern University, Feinberg School of Medicine, Chicago, IL, USA & RCT & 40 & $40(100.0)$ & $12[\mathrm{NA}]$ & $0(0.0)$ & $0(0.0)$ & $0(0.0)$ & $0(0.0)$ & $0(0.0)$ & $0(0.0)$ & $0(0.0)$ & $0(0.0)$ & $0(0.0)$ & $0(0.0)$ \\
\hline Mueller et al. (26) & The Loyola University Chicago, Stritch School of Medicine, Maywood, IL, USA & RS & 226 & $181(80.0)$ & $3.25[0.2-67]$ & NA & NA & NA & NA & $26(14.3)$ & NA & NA & NA & NA & $4(15.3)$ \\
\hline Nosti et al. (27) & $\begin{array}{l}\text { FPMRS Medstar Washington Hospital Center, Georgetown University School of Medicine, } \\
\text { Washington, DC, USA }\end{array}$ & RS & 262 & $262(100.0)$ & $8[\mathrm{NA}]$ & NA & NA & NA & NA & $45(17.1)$ & NA & NA & NA & NA & NA \\
\hline Geller et al. (28) & University of North Carolina at Chapel Hill, Chapel Hill, NC, USA & PS & 28 & $28(100.0)$ & $12[\mathrm{NA}]$ & $0(0.0)$ & $0(0.0)$ & $1(50.0)$ & $1(50.0)$ & $2(7.14)$ & $2(100.0)$ & $0(0.0)$ & $0(0.0)$ & $0(0.0)$ & $2(100.0)$ \\
\hline Biler et al. (30) & University of Health Sciences Tepecik Training and Research Hospital, Izmir, Turkey & PS & 20 & $20(100.0)$ & $16[10-36]$ & $0(0.0)$ & $0(0.0)$ & $0(0.0)$ & $0(0.0)$ & $1(5.0)$ & $0(0.0)$ & $0(0.0)$ & $0(0.0)$ & $0(0.0)$ & $0(0.0)$ \\
\hline Xylinas et al. (32) & CHU Henri Mondor, Créteil, France & RS & 12 & $12(100.0)$ & 19.1 [8-28] & $0(0.0)$ & $0(0.0)$ & $0(0.0)$ & $0(0.0)$ & $0(0.0)$ & $0(0.0)$ & $0(0.0)$ & $0(0.0)$ & $0(0.0)$ & $0(0.0)$ \\
\hline Ploumidis et al. (33) & OLV Vattikuti Robotic Surgery Institute, Aalst, Belgium & RS & 95 & $95(100.0)$ & $14.8[19-49]$ & $2(50.0)$ & $0(0.0)$ & $1(25.0)$ & $1(25.0)$ & $4(4.21)$ & NA & NA & NA & NA & NA \\
\hline Barboglio et al. (37) & Dartmouth-Hitchcock Medical Center, Lebanon, NH, USA & PS & 127 & $92(72.4)$ & $12[\mathrm{NA}]$ & $7(100.0)$ & $0(0.0)$ & $0(0.0)$ & $0(0.0)$ & $7(7.60)$ & $2(100.0)$ & $0(0.0)$ & $0(0.0)$ & $0(0.0)$ & $2(28.5)$ \\
\hline Cucinella et al. (38) & "Villa Sofia-Cervello" Hospital, University of Palermo, Palermo, Italy & $\mathrm{RCT}$ & 20 & $20(100.0)$ & $6[\mathrm{NA}]$ & $0(0.0)$ & $0(0.0)$ & $0(0.0)$ & $0(0.0)$ & $0(0.0)$ & $0(0.0)$ & $0(0.0)$ & $0(0.0)$ & $0(0.0)$ & $0(0.0)$ \\
\hline Tan-Kim et al. (40) & University of California, San Diego, CA, USA & RS & 43 & $40(93.0)$ & $6.25 \pm 5.75$ & $1(33.3)$ & $0(0.0)$ & $2(66.6)$ & $0(0.0)$ & $3(7.5)$ & NA & NA & NA & NA & NA \\
\hline Di Marco et al. (42) & Mayo Clinic, Rochester, MN, USA & RS & 5 & $5(100.0)$ & $4[\mathrm{NA}]$ & $0(0.0)$ & $0(0.0)$ & $0(0.0)$ & $0(0.0)$ & $0(0.0)$ & $0(0.0)$ & $0(0.0)$ & $0(0.0)$ & $0(0.0)$ & $0(0.0)$ \\
\hline Zhao and Martin (43) & University of Alberta Edmonton, $\mathrm{AB}$, Canada & RS & 47 & $47(100.0)$ & NA & $0(0.0)$ & $0(0.0)$ & $5(71.4)$ & $2(28.5)$ & $7(14.8)$ & NA & NA & NA & NA & NA \\
\hline Akl et al. (44) & Mayo Clinic, Phoenix, AZ, USA & RS & 80 & $80(100.0)$ & NA & $1(33.3)$ & $1(33.3)$ & $1(33.3)$ & $0(0.0)$ & $3(3.75)$ & 2 (66.6) & $1(33.3)$ & $0(0.0)$ & $0(0.0)$ & $3(100.0)$ \\
\hline Antosh et al. (45) & Washington Hospital Center, Washington, DC, USA & RS & 65 & $65(100.0)$ & $3[\mathrm{NA}]$ & $0(0.0)$ & $0(0.0)$ & $0(0.0)$ & $0(0.0)$ & $0(0.0)$ & NA & NA & NA & NA & NA \\
\hline Salamon and Culligan (47) & Division of Urogynecology, Atlantic Health System, Morristown, NJ, USA & RS & 64 & $64(100.0)$ & $12[\mathrm{NA}]$ & $3(50.0)$ & $1(16.6)$ & $2(33.3)$ & $0(0.0)$ & $6(9.37)$ & NA & NA & NA & NA & NA \\
\hline Culligan et al. (48) & Atlantic Health System, Morristown, NJ, USA & PS & 150 & $149(99.3)$ & $12[\mathrm{NA}]$ & $3(50.0)$ & $1(16.6)$ & $2(33.3)$ & $0(0.0)$ & $6(4.02)$ & NA & NA & NA & NA & NA \\
\hline Benson et al. (49) & Southern Illinois University, Carbondale, IL, USA & RS & 33 & $33(100.0)$ & $38.4[\mathrm{NA}]$ & $0(0.0)$ & $1(100.0)$ & $0(0.0)$ & $0(0.0)$ & $1(3.03)$ & NA & NA & NA & NA & NA \\
\hline Siddiqui et al. (50) & Duke University Medical Center, Durham, NC, USA & RS & 125 & $84(67.2)$ & NA & $0(0.0)$ & $0(0.0)$ & $3(100.0)$ & $0(0.0)$ & $3(3.57)$ & NA & NA & NA & NA & $3(100.0)$ \\
\hline Mourik et al. (52) & Maasstad Hospital, Rotterdam, Netherlands & PS & 50 & $50(100.0)$ & 16 [8-29] & NA & NA & NA & NA & $1(2.00)$ & $1(100.0)$ & $0(0.0)$ & $0(0.0)$ & $0(0.0)$ & $1(100.0)$ \\
\hline Overall & - & - & 2,029 & 1,852 (91.2) & $12[0.2-114]$ & $26(18.9)$ & $7(5.10)$ & $24(17.5)$ & $4(2.91)$ & $137(7.39)$ & $15(60.0)$ & $1(4.0)$ & $0(0.0)$ & $2(8.0)$ & $25(18.2)$ \\
\hline
\end{tabular}


conversions $(15 \%)$ from robotic to laparoscopy and 35 $(85 \%)$ to open surgery were recorded $(8,11,13,15,17$ $20,23,24,27,29,43,52,57)$. The conversions to LSC were due to adhesions $[\mathrm{n}=1(16.7 \%)]$, robot malfunctions $[\mathrm{n}=2$ $(33.3 \%)]$, and technical problems that were related to the robot $[\mathrm{n}=3(50 \%)]$. The cases of RSC converted to abdominal sacrocolpopexy (ASC) were due to difficult exposure or adhesions [n=26 (76.5\%)], pneumoperitoneum intolerance $[\mathrm{n}=1(2.9 \%)]$, obesity $[\mathrm{n}=1(2.9 \%)]$, vascular injury $[\mathrm{n}=1(2.9 \%)]$, cystotomies $[\mathrm{n}=1(2.9 \%)]$, cystotomies and injury to the sigmoid colon $[\mathrm{n}=1(2.9 \%)]$, and other complications $[\mathrm{n}=3(8.8 \%)]$.

\section{Cure and recurrence rate}

A summary of the recurrence rate in the RSC series is presented in Table 4. We only summarized the objective rather than subjective cure rates due to the different studies assessed outcomes considering different variables, thus making the subjective results between studies of no comparative significance. Thirty-four studies recorded 2,029 RSC patients' treatment outcomes, and 1,852 (91.3\%) RSC patients have been followed up, with a median postoperative follow-up duration of 12 [1-62] months. Overall, we observed that 134 of 1,852 patients $(7.2 \%)$ had recurred prolapses of any compartment (POP-Q $\geq$ grade 2) according to gynecological examination results, and RSC ensured a cumulative cure rate that ranged from $82.35-100 \%$. There were 26 RSC patients (19.4\%) with recurrence in the anterior compartment, $24(17.9 \%)$ in the posterior compartment, $4(3.0 \%)$ in both the anterior and posterior compartments, and 7 (5.2\%) with recurred apical prolapse. Although the cure rate of apical prolapse was higher than that of anterior and posterior recurrent prolapse, ranging from $96.7 \%$ to $100 \%$, there were 77 (57.5\%) RSC patients without records of the type of recurred prolapses. Also, few studies had a long follow-up duration of over 24 months, so determining the actual recurrence rate of apical prolapse needs more long-term follow-up results. A total of 25 patients $(18.7 \%)$ had been reported to reoperation because of prolapse. The surgeons almost always recommended recurrent prolapse patients to undergo reoperation through vaginal colporrhaphy or sacrocolpopexy $[60 \%(n=15)]$. Only 1 (4\%) and 2 patients (8\%) had undergone RSC and ASC, respectively. The participants who underwent reoperation through RSC and ASC were those who had recurred apical prolapse.

\section{Robot-assisted sacrocolpopexy compared with the laparoscopic approach}

\section{Intraoperative outcomes and complications}

The intraoperative outcomes are summarized in Figure 2 and Table S1. A total of $9(14,16,23,26,35,36,38,40,57)$ studies reported intraoperative blood loss. The results showed that the intraoperative blood loss in the RSC group was significantly less than that in the LSC group $(\mathrm{WMD}=-58.48 \mathrm{~mL}, 95 \% \mathrm{CI}:-100.58$ to -16.39 , $\mathrm{P}=0.006)$ with a high heterogeneity $\left(\mathrm{P}<0.00001, \mathrm{I}^{2}=98 \%\right)$ (Figure $2 A)$. A total of $11(6,15,16,23,26,29,35,36,38$, $40,57)$ studies reported the operation times, which revealed a significant difference between RSC and LSC $(\mathrm{WMD}=37.35$ minutes, $95 \% \mathrm{CI}: 24.46$ to 50.24 , $\mathrm{P}<0.00001)$ with a high heterogeneity $\left(\mathrm{P}<0.00001, \mathrm{I}^{2}=82 \%\right)$ (Figure 2B). A total of $8(14,23,26,29,44,57-59)$ studies reported the rate of conversion, and the results showed that there was much lower conversion rate in RSC than that in $\mathrm{LSC}(\mathrm{OR}=0.35,95 \% \mathrm{CI}: 0.15$ to $0.79, \mathrm{P}=0.01)$ without significant statistical heterogeneity $\left(\mathrm{P}=0.51, \mathrm{I}^{2}=0 \%\right)$ (Figure $2 C$ ).

A total of $13(6,14-16,23,26,29,36,40,44,57-59)$ studies reported intraoperative complications. The meta-analysis showed no statistically significant difference in the rate of intraoperative complications between RSC and the LSC (pooled $\mathrm{OR}=0.70,95 \% \mathrm{CI}$ : 0.46 to $1.06, \mathrm{P}=0.09$ ) without significant statistical heterogeneity $\left(\mathrm{P}=0.4, \mathrm{I}^{2}=4 \%\right)$ (Figure 2D). Among them, $10(6,15,16,26,29,36,40,44,58,59)$ studies reported intraoperative bladder injury, $9(6,15,16,26,29,36,40,58,59)$ studies reported intraoperative intestinal damage, $6(6,16,29,36,44,59)$ studies reported intraoperative vascular injury, and $5(6,16,26,29,59)$ studies reported intraoperative ureteral injury, respectively. Similarly, the results showed no statistical significance in the rate of intraoperative bladder injury (RSC $2.2 \%$ vs. LSC $3.1 \%$, $\mathrm{P}=0.17$ ), rate of intraoperative intestinal damage (RSC $0.6 \% v s$. LSC $1.1 \%, \mathrm{P}=0.43$ ), rate of intraoperative vascular injury (RSC $0.8 \%$ vs. LSC $0.8 \%, \mathrm{P}=0.66)$, and the rate of intraoperative ureteral injury (RSC $0.2 \%$ vs. LSC $0.0 \%, \mathrm{P}=0.41$ ) (Figure $2 E, F, G, H)$.

\section{Postoperative outcomes and complications}

The postoperative outcomes are summarized in Figure 3 and Table S2. Length of hospital stay was reported in $9(14-16,23,38,40,44,57,59)$ studies. There was no significant differences in length of hospital stay between the RSC and the LSC (WMD $=0.31,95 \%$ CI: -0.64 


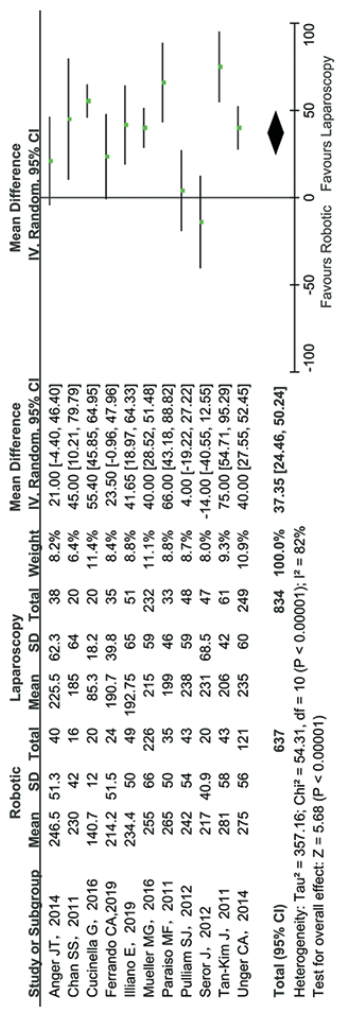

$\infty$

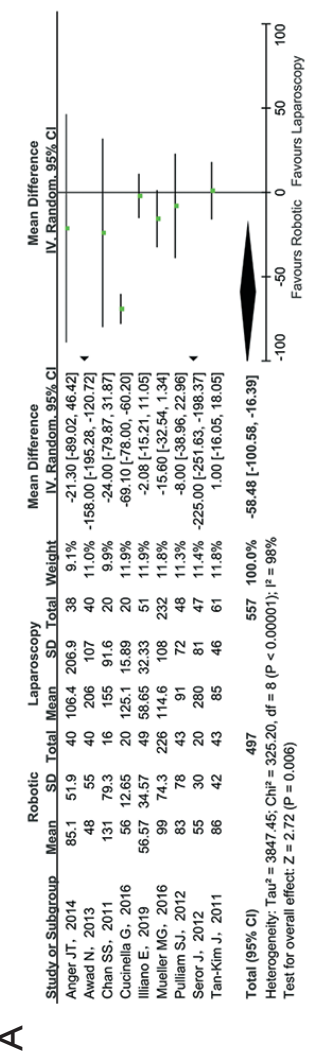

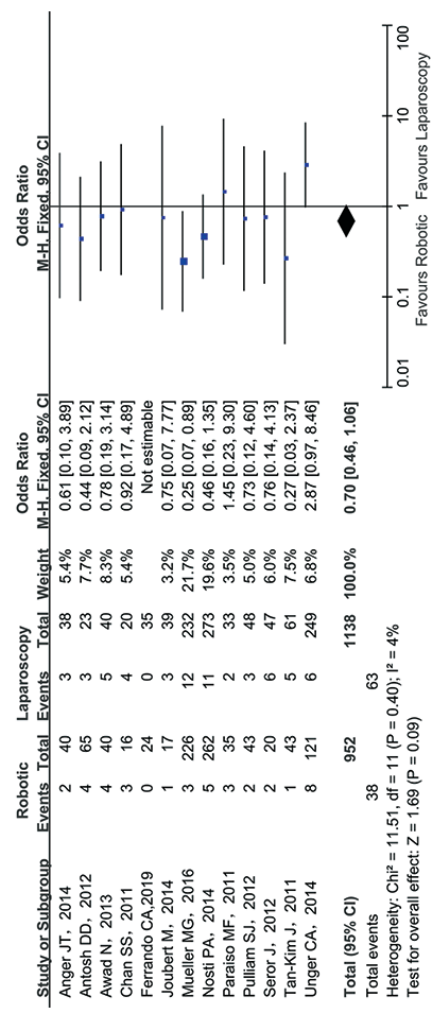
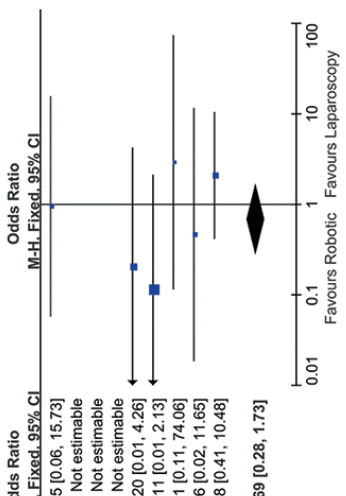

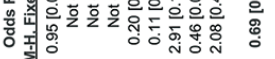

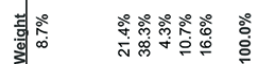

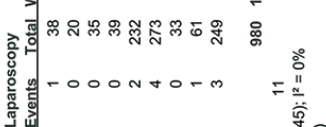

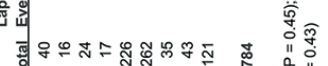

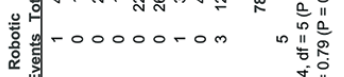

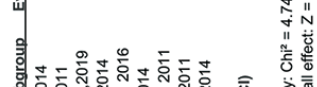

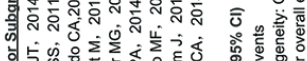

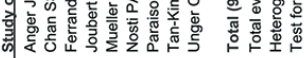

ᄂ

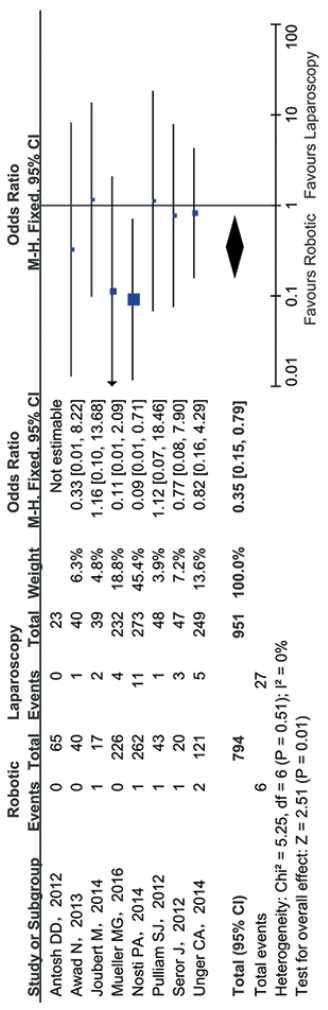

$\cup$

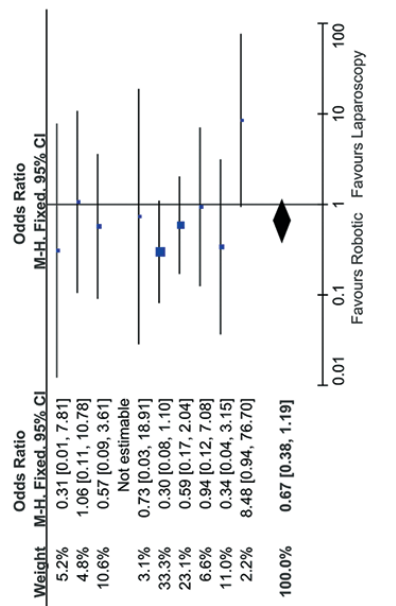

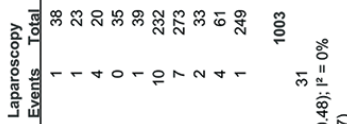

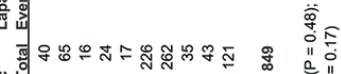

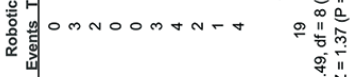

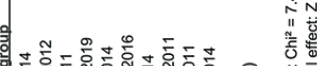

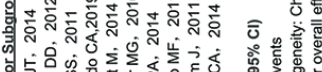

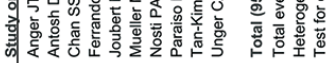

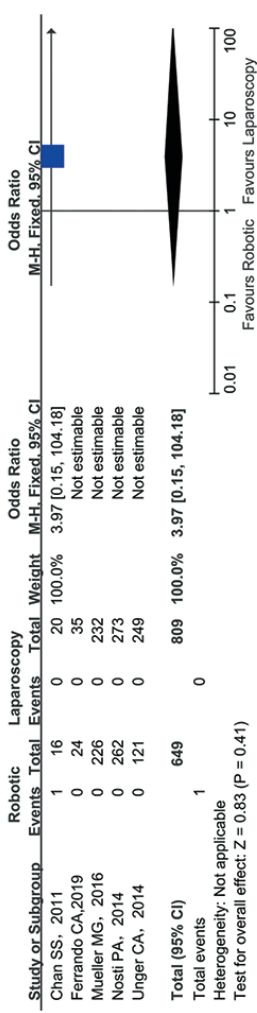

工

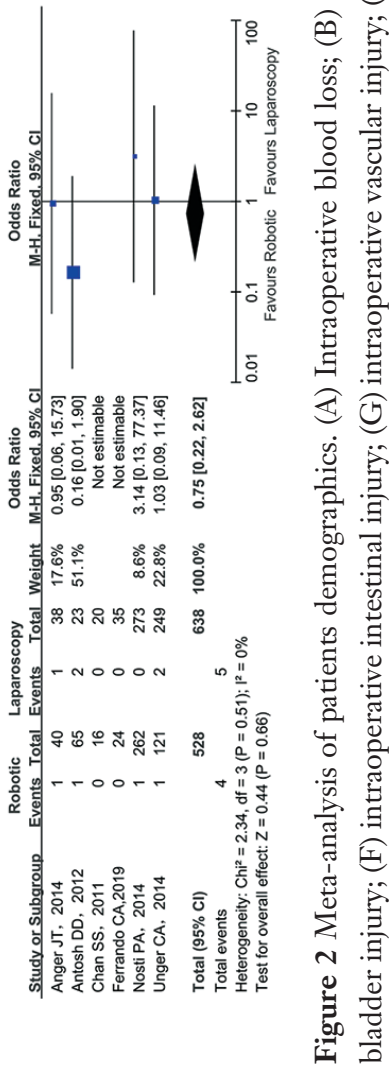




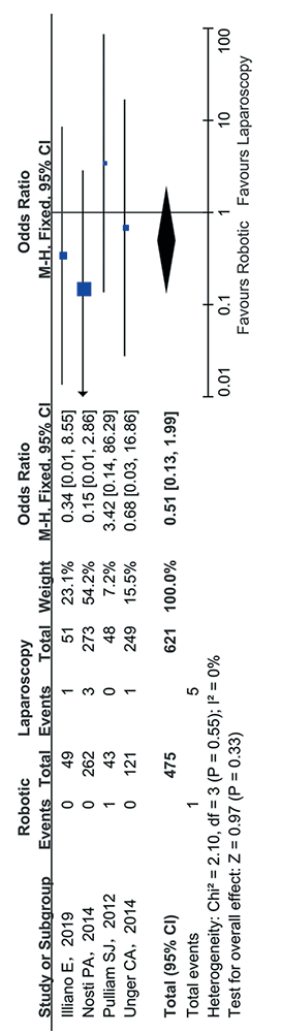

$\infty$

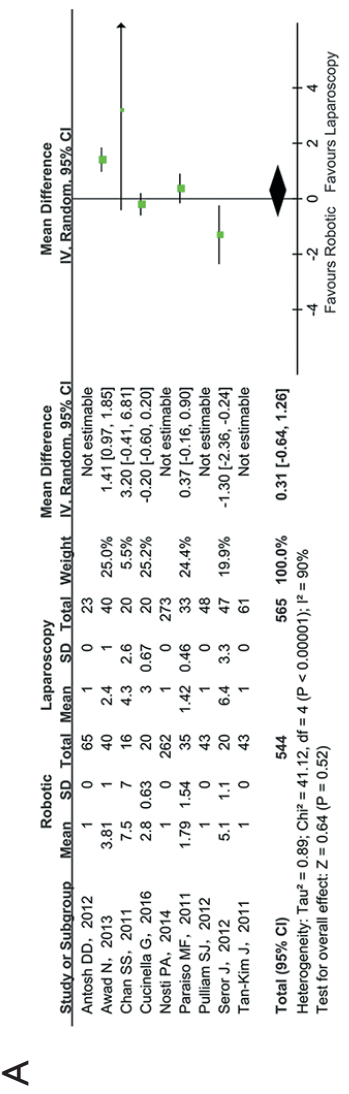

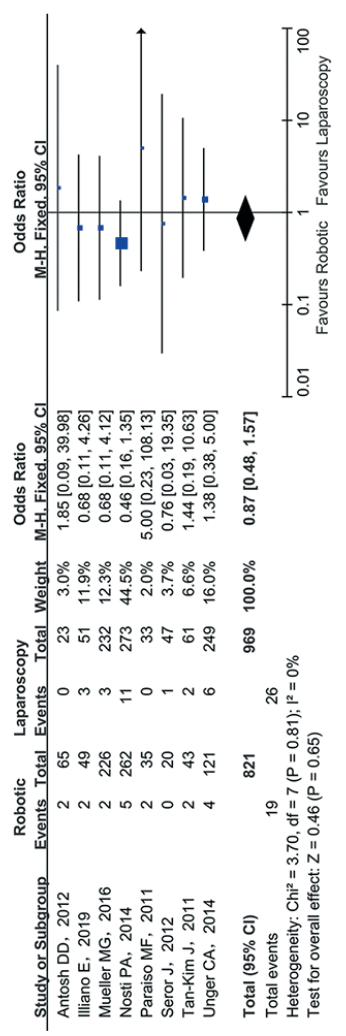

口

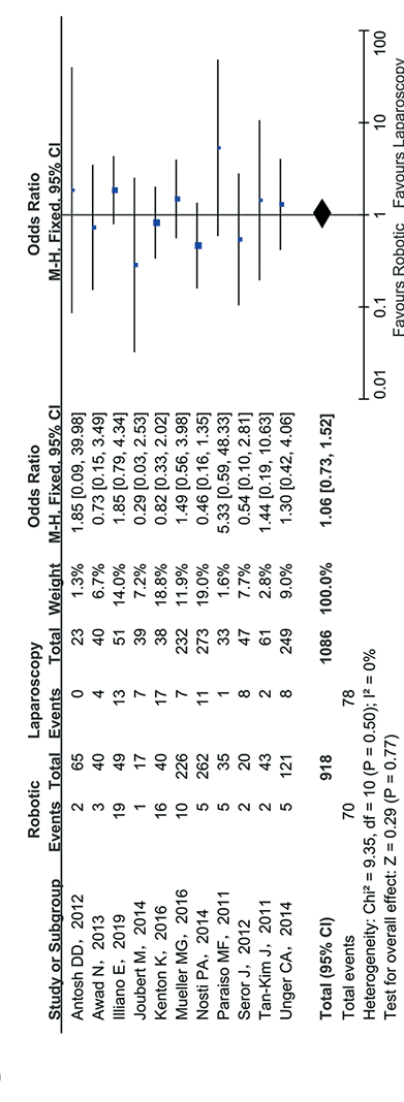

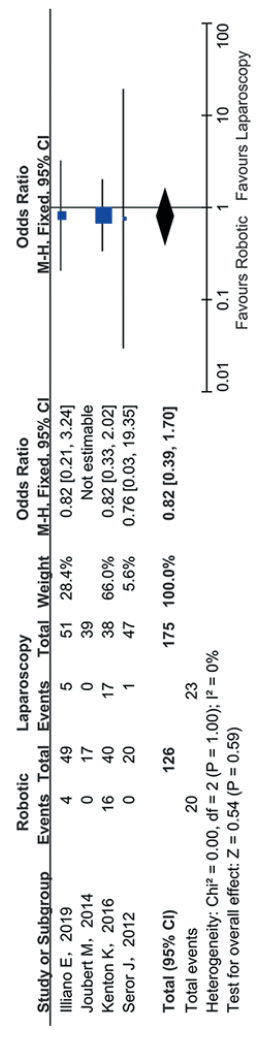

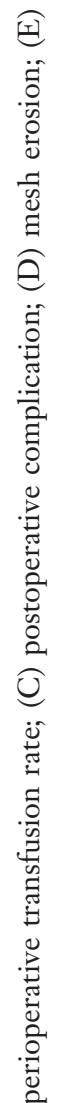

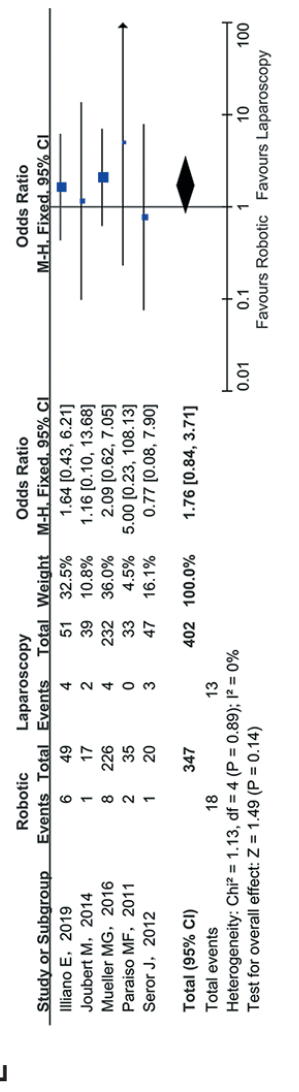


to $1.26, \mathrm{P}=0.52)$ with a high heterogeneity $(\mathrm{P}<0.00001$, $\mathrm{I}^{2}=90 \%$ ) (Figure $3 A$ ). Perioperative transfusion was reported in $4(23,29,35,59)$ studies. Similarly, no significant differences were observed in perioperative transfusion between the RSC and the $\mathrm{LSC}(\mathrm{OR}=0.51$, $95 \% \mathrm{CI}: 0.13$ to $1.99, \mathrm{P}=0.33)$ without significant heterogeneity $\left(\mathrm{P}=0.55, \mathrm{I}^{2}=0 \%\right)$ (Figure $3 B$ ). A total of $11(14,15,25,26,29,35,40,44,57-59)$ studies reported overall and major postoperative complications, and the results showed no statistically significant difference in the rate of postoperative complications between the RSC and LSC $(\mathrm{OR}=1.06,95 \% \mathrm{CI}: 0.73$ to $1.52, \mathrm{P}=0.77)$ without significant heterogeneity $\left(\mathrm{P}=0.5, \mathrm{I}^{2}=0 \%\right)$ (Figure $\left.3 C\right)$. Of these, $8(15,26,29,35,40,44,57,59)$ studies reported the rate of erosion of the mesh, $5(15,26,35,57,58)$ reported postoperative anorectal dysfunction, and $4(25,35,57,58)$ reported postoperative sexual disorders, respectively. Results of meta-analysis showed that there was no significant difference in the rate of erosion of the mesh (RSC $2.3 \%$ vs. LSC $2.7 \%, \mathrm{P}=0.65)$, rate of postoperative anorectal dysfunction (RSC 5.2\% vs. LSC 3.2\%, P=0.14), and the rate of postoperative sexual disorders (RSC $15.9 \%$ vs. LSC $13.1 \%, \mathrm{P}=0.59$ ) (Figure 3D,E,F).

\section{Cure and recurrence}

The cure and recurrence outcomes are summarized in Figure 4 and Table S3. A total of $5(14-16,35,58)$ studies reported the cure rate of $\mathrm{POP}$ ( $\mathrm{POP} \leq 1$ grade), and the results showed that the difference between RSC and LSC in a cure rate of POP was not statistically significant $(\mathrm{OR}=1.30,95 \% \mathrm{CI}: 0.55$ to $3.05, \mathrm{P}=0.55)$ without significant heterogeneity $\left(\mathrm{P}=0.77, \mathrm{I}^{2}=0 \%\right)$ (Figure $\left.4 A\right)$. Objective recurrence was reported in $8(15,26,36,40,44,57-59)$ studies. There was no significant difference in the rate of objective recurrence between $\mathrm{RSC}$ and $\mathrm{LSC}(\mathrm{OR}=1.20$, 95\% CI: 0.83 to $1.73, \mathrm{P}=0.34)$ without significant heterogeneity $\left(\mathrm{I}^{2}=33 \%, \mathrm{P}=0.16\right)$ (Figure $\left.4 B\right)$. A total of $5(16,26,36,44,58)$ studies reported the reoperation rate, showing that the difference between RSC and LSC in reoperation rate was not statistically significant $(\mathrm{OR}=0.66,95 \% \mathrm{CI}: 0.27$ to 1.61 , $\mathrm{P}=0.36)$ without significant heterogeneity $\left(\mathrm{P}=0.9, \mathrm{I}^{2}=0 \%\right)$ (Figure 4C).

\section{Publications bias}

Egger's test was used to assess the publication bias for four outcomes. The $\mathrm{P}$ values for operative time, intraoperative blood loss, postoperative complication, conversion rate, and objective recurrence ( 24 months) were $0.206,0.765,0.865$,
0.309 , and 0.327 , respectively. No significant publication bias was discovered among the studies.

\section{Discussion}

In this systematic review and meta-analysis of 49 articles, including 3,014 patients, 18 were comparative studies on LSC $v s$. RSC, and 31 were non-comparative single-arm studies on RSC. For RSC, the median operative time was 226 [90-604] minutes, estimated blood loss was 56 [5-1,500] mL, and hospital stay was 1.55 [1-16] days. Intraoperative complications and postoperative complications occurred in $74(2.7 \%)$ and $360(13.0 \%)$ patients, respectively. Out of 2,768 RSC patients, 40 had been converted from a robot-assisted approach to other approaches, 134 of 1,852 patients $(7.2 \%)$ had recurred prolapses of any compartment. Compared with LSC, RSC was associated with significantly lower blood loss (WMD $=-58.48 \mathrm{~mL}, 95 \% \mathrm{CI}:-100.58$ to $-16.39, \mathrm{P}=0.006)$ and lower conversion rate $(\mathrm{OR}=0.35,95 \%$ CI: 0.15 to $0.79, \mathrm{P}=0.01)$. However, longer operative time (WMD $=37.35$ minutes, $95 \%$ CI: 24.46 to $50.24, \mathrm{P}<0.00001$ ) and no significant difference in perioperative transfusion, intraoperative and postoperative complications, and objective recurrence were observed between RSC and LSC.

Sacrocolpopexy is the "gold standard" procedure for the treatment of POP patients with apical defects. Different surgical approaches have emerged successively over recent years, including traditional laparoscopy, single-hole laparoscopy, robot-assisted laparoscopy, vaginal-assisted laparoscopy, and transvaginal approaches, with different surgical approaches having different respective advantages (60). The LSC has become the current mainstream approach of this operation due to its advantages of minimal trauma, high cure rate, and low recurrence rate (3). However, from analysis of the anatomical structure, the anterior longitudinal ligament on the pelvic surface of S1 vertebra in the presacral region is a relatively safe suture area for sacral fixation (the upper boundary is $10 \mathrm{~mm}$ below the promontory, the lower boundary is $40 \mathrm{~mm}$ below the promontory, and the horizontal width is $15 \mathrm{~mm}$ ) (61). LSC operation is mainly performed in this area, but the sacrococcygeal curvature in this area is concave backward, which is a relatively "blind area" for laparoscopic surgery. Besides, the presacral region's anatomy is complex, rich in blood vessels, and has high anatomical variability. Considering that the right internal iliac vein is a large vessel nearby and the presacral venous plexus is interwoven into a mesh, it is challenging to arrest bleeding following injury to the presacral vessels and bleeding can easily occur during 


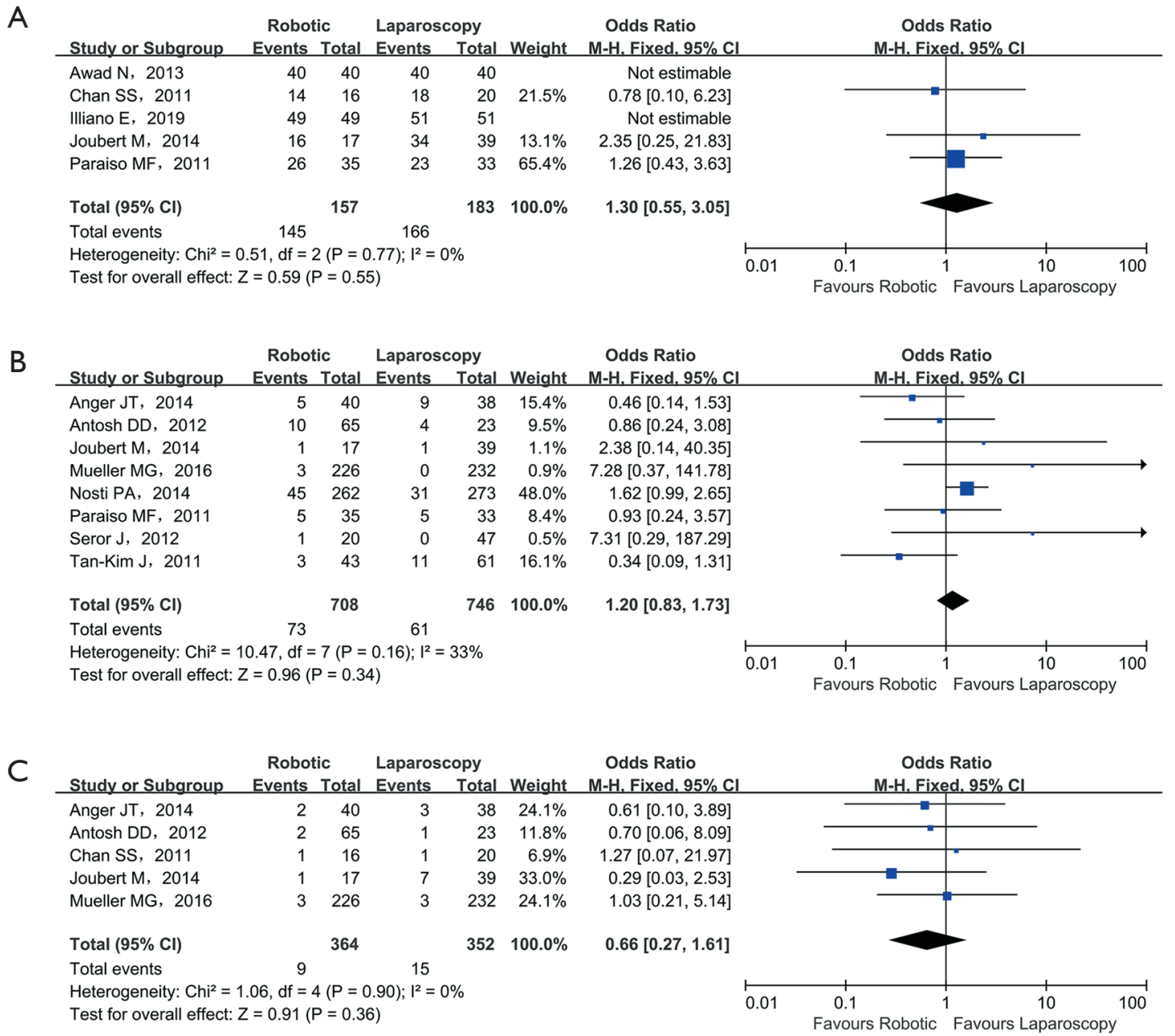

Figure 4 Meta-analysis of patients demographics. (A) Cure rate of POP; (B) objective recurrence (24-month); (C) reoperation rate. POP, pelvic organ prolapse; CI, confidence interval.

the operation. In addition, the pelvic cavity is occupied by uterus, ovaries, bladder, rectum, and other organs, and the ureter travels on both sides; thus, it is difficult to surgically separate and suture $(62,63)$. Consequently, the principal limitations of traditional LSC are the difficulty of suturing and the risk of vascular injury provoked by the chopstick effect of two-dimensional (2D) vision and instruments.

The RSC has attracted increasing attention as an emerging surgical technique with unique advantages (64). Firstly, the robotic surgery system's camera has a duallens structure, which provides doctors with a super-clear, high-fold surgical field, and 3D visualization of the pelvis. Secondly, its mechanical arm has "7 degrees of freedom" and installs a flutter filter device, enabling the simulated wrist to rotate $540^{\circ}$, with the unique properties of high precision, flexibility, and stability. Thirdly, the console is designed according to the mechanical characteristics of the human body. By controlling the handle and pedal, the operator can realize the precise real-time movement of the wrist, hand, and fingers through the sensing system, allowing operator comfort and diminishing operator fatigue. Fourthly, it circumvents the disadvantage of poor cooperation between the operator and assistant that inevitably appears during LSC (64). Da Vinci robotic surgery was successfully applied in gynecological surgery for the first time in 2004, and it was officially approved by the Food and Drug Administration (FDA) for use in the gynecological clinic in 2005 .

Intraoperative bleeding volume is a crucial parameter to evaluate the quality of surgery, and it is significantly 
related to the recovery of patients after surgery. Our results demonstrated that the intraoperative bleeding volume of the RSC group was significantly lower than that of LSC; the maximum median blood loss of RSC was $131 \mathrm{~mL}$, in comparison, that of LSC was $280 \mathrm{~mL}$; there were 5 out of 621 LSC series that required transfusion, and just 1 out of 475 participants in RSC, which indicated that robotic surgery had apparent advantages in meticulous and precise operation. The RSC also revealed significant advantages in avoiding conversion of laparoscopic surgery. In essence, RSC's conversion rate was $0.76 \%$ (7/794), mainly due to severe pelvic and abdominal adhesions, while the conversion rate of LSC was 2.8\% (27/951); besides adhesions, bladder and bowel injury were the main reasons leading to conversion. In comparing the operation time, that of the RSC group was longer while a broad range of operation time was seen among different institutions, which may be generated by the following reasons. First, there was no uniform criterion to measure operation time. Some hospitals had included the docking time of the robot surgical system, which adds an extra 3-60 mins (15). Besides, some data were collected during the early adoption phase for RSC at numerous institutions. Additionally, surgeons' experience always has an inverse correlation with the duration of surgery. Akl and Awad et al. reported that operative time decreased $>25 \%$ after the execution of the first 10 cases, which was primarily attributed to the shortening of console time $(14,43)$. Also, Geller et al. described that time of cuff closure, anterior and posterior sacral dissection, sacral mesh attachment, peritoneal closure, total docked time, and total incision time decreased after the first 20 procedures of RSC, which suggested that the learning curve of RSC is an influential factor on operative time (7). In comparing perioperative complications, average hospitalization days, post-operative cure rate, and objective recurrence rate, there were no significant differences between the two surgical methods, indicating that both methods had the characteristics of high cure rate and low recurrence rate in terms of clinical efficacy.

The principal disadvantage of the robotic surgery system is the inherent cost and high maintenance cost. However, advantages such as the "simulated wrist" mechanical arm and high-definition 3D visual field effectively overcome the problem of "blind area" of vision and operation in LSC surgery, which provides a positive guarantee for surgical operation. They can effectively reduce the risk of vascular injury, bleeding, and endoscopic transfer in the presacral area.

\section{Conclusions}

The RSC appears to offer some advantages compared to conventional laparoscopic surgery, although both approaches appear to offer equivalent clinical outcomes. It is crucial to note that heterogeneity among studies may have affected this study's outcome, and a high-quality and large-sample randomized trial comparing both techniques is required.

\section{Acknowledgments}

We gratefully acknowledge the contributions of numerous investigators that we were unable to include in this review, owing to space limitations. This research was sponsored by Hubei Province's Outstanding Medical Academic Leader Program. Part of the funds was used to gain access to the database.

Funding: This research is supported by Hubei Province's Outstanding Medical Academic Leader Program.

\section{Footnote}

Reporting Checklist: The authors have completed the PRISMA reporting checklist. Available at http://dx.doi. org/10.21037/atm-20-4347

Peer Review File: Available at http://dx.doi.org/10.21037/ atm-20-4347

Conflicts of Interest: All authors have completed the ICMJE uniform disclosure form (available at http://dx.doi. org/10.21037/atm-20-4347). The authors have no conflicts of interest to declare.

Ethical Statement: The authors are accountable for all aspects of the work in ensuring that questions related to the accuracy or integrity of any part of the work are appropriately investigated and resolved.

Open Access Statement: This is an Open Access article distributed in accordance with the Creative Commons Attribution-NonCommercial-NoDerivs 4.0 International License (CC BY-NC-ND 4.0), which permits the noncommercial replication and distribution of the article with the strict proviso that no changes or edits are made and the original work is properly cited (including links to both the formal publication through the relevant DOI and the license). 
See: https://creativecommons.org/licenses/by-nc-nd/4.0/.

\section{References}

1. Peng P, Zhu L, Lang JH, et al. Unilateral sacrospinous ligament fixation for treatment of genital prolapse. Chin Med J (Engl) 2010;123:1995-8.

2. Luber KM, Boero S, Choe JY. The demographics of pelvic floor disorders: current observations and future projections. Am J Obstet Gynecol 2001;184:1496-501; discussion 1501-3.

3. Ganatra AM, Rozet F, Sanchez-Salas R, et al. The current status of laparoscopic sacrocolpopexy: a review. Eur Urol 2009;55:1089-103.

4. Aromataris E, Munn Z. editors. JBI manual for evidence synthesis. JBI, 2020. Available online: https:// synthesismanual.jbi.global

5. Slim K, Nini E, Forestier D, et al. Methodological index for non-randomized studies (minors): development and validation of a new instrument. ANZ J Surg 2003;73:712-6.

6. Ferrando CA, Paraiso MFR. A prospective randomized trial comparing restorelle y mesh and flat mesh for laparoscopic and robotic-assisted laparoscopic sacrocolpopexy. Female Pelvic Med Reconstr Surg 2019;25:83-7.

7. Geller EJ, Lin FC, Matthews CA. Analysis of robotic performance times to improve operative efficiency. J Minim Invasive Gynecol 2013;20:43-8.

8. Elliott DS, Siddiqui SA, Chow GK. Assessment of the durability of robot-assisted laparoscopic sacrocolpopexy for treatment of vaginal vault prolapse. J Robot Surg 2007;1:163-8.

9. Mueller ER, Kenton K, Anger JT, et al. Cosmetic appearance of port-site scars 1 year after laparoscopic versus robotic sacrocolpopexy: a supplementary study of the ACCESS clinical trial. J Minim Invasive Gynecol 2016;23:917-21.

10. Elliott DS, Chow GK, Gettman M. Current status of robotics in female urology and gynecology. World J Urol 2006;24:188-92.

11. Shariati A, Maceda JS, Hale DS. Da Vinci assisted laparoscopic sacrocolpopexy. J Pelvic Med Surg 2008;14:163-71.

12. Collins SA, Tulikangas PK, O'Sullivan DM. Effect of surgical approach on physical activity and pain control after sacral colpopexy. Am J Obstet Gynecol 2012;206:438.e1-6.
13. Elliott DS, Frank I, Dimarco DS, et al. Gynecologic use of robotically assisted laparoscopy: Sacrocolpopexy for the treatment of high-grade vaginal vault prolapse. Am J Surg 2004;188:52S-6S.

14. Awad N, Mustafa S, Amit A, et al. Implementation of a new procedure: laparoscopic versus robotic sacrocolpopexy. Arch Gynecol Obstet 2013;287:1181-6.

15. Paraiso MF, Jelovsek JE, Frick A, et al. Laparoscopic compared with robotic sacrocolpopexy for vaginal prolapse: a randomized controlled trial. Obstet Gynecol 2011;118:1005-13.

16. Chan SSC, Pang SMW, Cheung TH, et al. Laparoscopic sacrocolpopexy for the treatment of vaginal vault prolapse: with or without robotic assistance. Hong Kong Med J 2011;17:54-60.

17. Moreno Sierra J, Ortiz Oshiro E, Fernandez Perez C, et al. Long-term outcomes after robotic sacrocolpopexy in pelvic organ prolapse: prospective analysis. Urol Int 2011;86:414-8.

18. Shimko MS, Umbreit EC, Chow GK, et al. Long-term outcomes of robotic-assisted laparoscopic sacrocolpopexy with a minimum of three years follow-up. J Robot Surg 2011;5:175-80.

19. Linder BJ, Chow GK, Elliott DS. Long-term quality of life outcomes and retreatment rates after robotic sacrocolpopexy. Int J Urol 2015;22:1155-8.

20. Elliott DS, Krambeck AE, Chow GK. Long-term results of robotic assisted laparoscopic sacrocolpopexy for the treatment of high grade vaginal vault prolapse. J Urol 2006;176:655-9.

21. Osmundsen BC, Clark A, Goldsmith C, et al. Mesh erosion in robotic sacrocolpopexy. Female Pelvic Med Reconstr Surg 2012;18:86-8.

22. Belsante M, Murray S, Dillon B, et al. Mid term outcome of robotic mesh sacrocolpopexy. Can J Urol 2013;20:6656-61.

23. Pulliam SJ, Weinstein MM, Wakamatsu MM. Minimally invasive apical sacropexy: a retrospective review of laparoscopic and robotic operating room experiences. Female Pelvic Med Reconstr Surg 2012;18:122-6.

24. Jambusaria LH, Murphy M, Lucente VR. One-year functional and anatomic outcomes of robotic sacrocolpopexy versus vaginal extraperitoneal colpopexy with mesh. Female Pelvic Med Reconstr Surg 2015;21:87-92.

25. Kenton K, Mueller ER, Tarney C, et al. One-year outcomes after minimally invasive sacrocolpopexy. Female Pelvic Med Reconstr Surg 2016;22:382-4.

26. Mueller MG, Jacobs KM, Mueller ER, et al. Outcomes in 450 women after minimally invasive abdominal 
sacrocolpopexy for pelvic organ prolapse. Female Pelvic Med Reconstr Surg 2016;22:267-71.

27. Nosti PA, Umoh Andy U, Kane S, et al. Outcomes of abdominal and minimally invasive sacrocolpopexy: a retrospective cohort study. Female Pelvic Med Reconstr Surg 2014;20:33-7.

28. Geller EJ, Parnell BA, Dunivan GC. Pelvic floor function before and after robotic sacrocolpopexy: one-year outcomes. J Minim Invasive Gynecol 2011;18:322-7.

29. Unger CA, Paraiso MF, Jelovsek JE, et al. Perioperative adverse events after minimally invasive abdominal sacrocolpopexy. Am J Obstet Gynecol 2014;211:547.e1-8.

30. Biler A, Ertas IE, Tosun G, et al. Perioperative complications and short-term outcomes of abdominal sacrocolpopexy, laparoscopic sacrocolpopexy, and laparoscopic pectopexy for apical prolapse. Int Braz J Urol 2018;44:996-1004.

31. Matthews CA, Carroll A, Hill A, et al. Prospective evaluation of surgical outcomes of robot-assisted sacrocolpopexy and sacrocervicopexy for the management of apical pelvic support defects. South Med J 2012;105:274-8.

32. Xylinas E, Ouzaid I, Durand X, et al. Robot-assisted laparoscopic sacral colpopexy: initial experience in a high-volume laparoscopic reference center. J Endourol 2010;24:1985-9.

33. Ploumidis A, Spinoit AF, De Naeyer G, et al. Robotassisted sacrocolpopexy for pelvic organ prolapse: surgical technique and outcomes at a single high-volume institution. Eur Urol 2014;65:138-45.

34. Elliott CS, Hsieh MH, Sokol ER, et al. Robot-assisted versus open sacrocolpopexy: a cost-minimization analysis. J Urol 2012;187:638-43.

35. Illiano E, Ditonno P, Giannitsas K, et al. Robot-assisted vs laparoscopic sacrocolpopexy for high-stage pelvic organ prolapse: a prospective, randomized, single-center study. Urology 2019;134:116-23.

36. Anger JT, Mueller ER, Tarnay C, et al. Robotic compared with laparoscopic sacrocolpopexy: a randomized controlled trial. Obstet Gynecol 2014;123:5-12.

37. Barboglio PG, Toler AJ, Triaca V. Robotic sacrocolpopexy for the management of pelvic organ prolapse: a review of midterm surgical and quality of life outcomes. Female Pelvic Med Reconstr Surg 2014;20:38-43.

38. Cucinella G, Calagna G, Romano G, et al. Robotic versus laparoscopic sacrocolpopexy for apical prolapse: a casecontrol study. G Chir 2016;37:113-7.

39. Geller EJ, Parnell BA, Dunivan GC. Robotic vs abdominal sacrocolpopexy: 44-month pelvic floor outcomes. Urology 2012;79:532-6.

40. Tan-Kim J, Menefee SA, Luber KM, et al. Robotic-assisted and laparoscopic sacrocolpopexy: comparing operative times, costs and outcomes. Female Pelvic Med Reconstr Surg 2011;17:44-9.

41. Louis-Sylvestre C, Herry M. Robotic-assisted laparoscopic sacrocolpopexy for stage III pelvic organ prolapse. Int Urogynecol J 2013;24:731-3.

42. Zhao Y, St Martin B. Robotic-assisted laparoscopic sacrocolpopexy: initial Canadian experience. Can Urol Assoc J 2020;14:E257-63.

43. Akl MN, Long JB, Giles DL, et al. Robotic-assisted sacrocolpopexy: technique and learning curve. Surg Endosc 2009;23:2390-4.

44. Antosh DD, Grotzke SA, McDonald MA, et al. Shortterm outcomes of robotic versus conventional laparoscopic sacral colpopexy. Female Pelvic Med Reconstr Surg 2012;18:158-61.

45. Matanes E, Lauterbach R, Mustafa-Mikhail S, et al. Single port robotic assisted sacrocolpopexy: our experience with the first 25 cases. Female Pelvic Med Reconstr Surg 2017;23:e14-8.

46. Salamon CG, Culligan PJ. Subjective and objective outcomes 1 year after robotic-assisted laparoscopic sacrocolpopexy. J Robot Surg 2013;7:35-8.

47. Culligan PJ, Gurshumov E, Lewis C, et al. Subjective and objective results 1 year after robotic sacrocolpopexy using a lightweight Y-mesh. Int Urogynecol J 2014;25:731-5.

48. Benson AD, Kramer BA, Wayment RO, et al. Supracervical robotic-assisted laparoscopic sacrocolpopexy for pelvic organ prolapse. JSLS 2010;14:525-30.

49. Siddiqui NY, Geller EJ, Visco AG. Symptomatic and anatomic 1-year outcomes after robotic and abdominal sacrocolpopexy. Am J Obstet Gynecol 2012;206:435.e1-5.

50. Bedaiwy MA, Abdelrahman M, Deter S, et al. The impact of training residents on the outcome of robotic-assisted sacrocolpopexy. Minim Invasive Surg 2012;2012:289342.

51. Mourik SL, Martens JE, Aktas M. Uterine preservation in pelvic organ prolapse using robot assisted laparoscopic sacrohysteropexy: quality of life and technique. Eur J Obstet Gynecol Reprod Biol 2012;165:122-7.

52. Thubert T, Dabi Y, Boudy AS, et al. Comparison of functional outcomes after robot-assisted laparoscopic sacrocolpopexy in women with a BMI below and above 30 . Urogynaecologia 2017. doi: 10.4081/uij.2017.178.

53. Borahay MA, Oge T, Walsh TM, et al. Outcomes of robotic sacrocolpopexy using barbed delayed absorbable 
sutures. J Minim Invasive Gynecol 2014;21:412-6.

54. Di Marco DS, Chow GK, Gettman MT, et al. Roboticassisted laparoscopic sacrocolpopexy for treatment of vaginal vault prolapse. Urology 2004;63:373-6.

55. Satava RM. Identification and reduction of surgical error using simulation. Minim Invasive Ther Allied Technol 2005;14:257-61.

56. Mitropoulos D, Artibani W, Graefen M, et al. Reporting and grading of complications after urologic surgical procedures: an ad hoc EAU guidelines panel assessment and recommendations. Eur Urol 2012;61:341-9.

57. Seror J, Yates DR, Seringe E, et al. Prospective comparison of short-term functional outcomes obtained after pure laparoscopic and robot-assisted laparoscopic sacrocolpopexy. World J Urol 2012;30:393-8.

58. Joubert M, Thubert T, Lefranc JP, et al. Comparison of functional outcomes with purely laparoscopic sacrocolpopexy and robot-assisted sacrocolpopexy in obese women. Prog Urol 2014;24:1106-13.

Cite this article as: Yang J, He Y, Zhang X, Wang Z, Zuo X, Gao L, Hong L. Robotic and laparoscopic sacrocolpopexy for pelvic organ prolapse: a systematic review and meta-analysis. Ann Transl Med 2021;9(6):449. doi: 10.21037/atm-20-4347
59. Nosti PA, Andy UU, Kane S, et al. Outcomes of abdominal and minimally invasive sacrocolpopexy: a retrospective cohort study. Female Pelvic Med Reconstr Surg 2014;20:33-7.

60. Costantini E, Brubaker L, Cervigni M, et al. Sacrocolpopexy for pelvic organ prolapse: evidence-based review and recommendations. Eur J Obstet Gynecol Reprod Biol 2016;205:60-5.

61. Barber MD. Pelvic organ prolapse. BMJ 2016;354:i3853.

62. Westerman ME, Elliott DS, Shimko MS, et al. Robotic sacrocolpopexy. In: Su LM. editor. Atlas of robotic urologic surgery. Cham: Springer, Cham, 2017:351-63.

63. Lee RK, Mottrie A, Payne CK, et al. A review of the current status of laparoscopic and robot-assisted sacrocolpopexy for pelvic organ prolapse. Eur Urol 2014;65:1128-37.

64. Watanabe G, Ishikawa N. da Vinci surgical system. Kyobu Geka 2014;67:686-9. 
Table S1 Summary of the meta-analysis regarding intraoperative outcomes and complications

\begin{tabular}{|c|c|c|c|c|c|c|c|}
\hline \multirow{2}{*}{ Outcomes } & \multirow{2}{*}{ No. of studies } & \multicolumn{2}{|c|}{ No. of patients } & \multirow{2}{*}{ OR/MD (95\% Cl) } & \multirow{2}{*}{$P$ value } & \multicolumn{2}{|c|}{ Tests for heterogeneity } \\
\hline & & RSC & LSC & & & $\mathrm{I}^{2}(\%)$ & $P$ \\
\hline Intraoperative blood loss (mL) & 9 & 497 & 557 & $-58.48(-100.58$ to -16.39$)$ & 0.006 & 98 & $<0.00001$ \\
\hline Operation time (min) & 11 & 637 & 834 & 37.35 (24.46 to 50.24 ) & $<0.00001$ & 82 & $<0.00001$ \\
\hline Conversion rate & 8 & 794 & 951 & 0.35 (0.15 to 0.79$)$ & 0.01 & 0 & 0.51 \\
\hline Intraoperative complication & 13 & 952 & 1,138 & $0.70(0.46$ to 1.06$)$ & 0.09 & 4 & 0.4 \\
\hline Intraoperative bladder injury & 10 & 849 & 1,003 & 0.67 (0.38 to 1.19$)$ & 0.17 & 0 & 0.48 \\
\hline Intraoperative intestinal injury & 9 & 784 & 980 & 0.69 (0.28 to 1.73$)$ & 0.43 & 0 & 0.45 \\
\hline Intraoperative vascular injury & 6 & 528 & 638 & 0.75 (0.22 to 2.62$)$ & 0.66 & 0 & 0.51 \\
\hline Intraoperative ureteral injury & 5 & 649 & 809 & $3.97(0.15$ to 104.18$)$ & 0.41 & 0 & 0 \\
\hline
\end{tabular}

LSC, laparoscopic sacrocolpopexy; RSC, robotic sacrocolpopexy; OR, odds ratio; MD, mean difference; $\mathrm{Cl}$, confidence interval.

Table S2 Summary of the meta-analysis regarding postoperative outcomes and complications

\begin{tabular}{|c|c|c|c|c|c|c|c|}
\hline \multirow{2}{*}{ Outcomes } & \multirow{2}{*}{ No. of studies } & \multicolumn{2}{|c|}{ No. of patients } & \multirow{2}{*}{ OR/MD (95\% Cl) } & \multirow{2}{*}{$P$ value } & \multicolumn{2}{|c|}{ Tests for heterogeneity } \\
\hline & & $\mathrm{RSC}$ & LSC & & & $I^{2}(\%)$ & $\mathrm{P}$ \\
\hline Length of hospital stay (d) & 9 & 544 & 565 & $0.31(-0.64$ to 1.26$)$ & 0.52 & 90 & $<0.00001$ \\
\hline Perioperative transfusion rate & 4 & 475 & 621 & 0.51 (0.13 to 1.99$)$ & 0.33 & 0 & 0.55 \\
\hline Postoperative complication & 11 & 918 & 1,086 & 1.06 (0.73 to 1.52$)$ & 0.77 & 0 & 0.5 \\
\hline Mesh erosion & 8 & 821 & 969 & $0.87(0.48$ to 1.57$)$ & 0.65 & 0 & 0.81 \\
\hline Postoperative anorectal dysfunction & 5 & 347 & 402 & $1.76(0.84$ to 3.71$)$ & 0.14 & 0 & 0.89 \\
\hline Postoperative sexual disorder & 4 & 126 & 175 & $0.82(0.39$ to 1.70$)$ & 0.59 & 0 & 1.00 \\
\hline
\end{tabular}

LSC, laparoscopic sacrocolpopexy; RSC, robotic sacrocolpopexy; OR, odds ratio; MD, mean difference; $\mathrm{Cl}$, confidence interval.

Table S3 Summary of the meta-analysis regarding cure and recurrence

\begin{tabular}{|c|c|c|c|c|c|c|c|}
\hline \multirow{2}{*}{ Outcomes } & \multirow{2}{*}{ No. of studies } & \multicolumn{2}{|c|}{ No. of patients } & \multirow{2}{*}{ OR/MD (95\% Cl) } & \multirow{2}{*}{$P$ value } & \multicolumn{2}{|c|}{ Tests for heterogeneity } \\
\hline & & RSC & LSC & & & $\mathrm{I}^{2}(\%)$ & $P$ \\
\hline Cure rate of POP & 5 & 157 & 183 & 1.30 (0.55 to 3.05$)$ & 0.55 & 0 & 0.77 \\
\hline Objective recurrence (24-month) & 8 & 708 & 746 & 1.20 (0.83 to 1.73$)$ & 0.34 & 33 & 0.16 \\
\hline Reoperation rate & 5 & 364 & 352 & $0.66(0.27$ to 1.61$)$ & 0.36 & 0 & 0.9 \\
\hline
\end{tabular}

LSC, laparoscopic sacrocolpopexy; RSC, robotic sacrocolpopexy; POP, pelvic organ prolapse; OR, odds ratio; MD, mean difference; Cl, confidence interval. 